\title{
Establishment of a 12-gene expression signature to predict colon cancer prognosis
}

\author{
Dalong Sun ${ }^{1}$, Jing Chen ${ }^{2}$, Longzi Liu ${ }^{3}$, Guangxi Zhao ${ }^{4}$ ， Pingping Dong ${ }^{1}$ ， Bingrui Wu ${ }^{5}$, Jun Wang ${ }^{\text {Corresp., }}{ }^{6}$, \\ Ling Dong ${ }^{\text {Corresp. } 1}$ \\ 1 Department of Gastroenterology and Hepatology, Zhongshan Hospital, Fudan University, Shanghai, China \\ 2 Department of Neurology, Shanghai Fifth People's Hospital, Fudan University, Shanghai, China \\ 3 Department of Hepatic Surgery, Liver Cancer Institute, and Key Laboratory of Carcinogenesis and Cancer Invasion (Ministry of Education), Zhongshan \\ Hospital, Fudan University, Shanghai, China \\ 4 Department of Gastroenterology, Shanghai East Hospital, Tongji University School of Medicine, Shanghai, China \\ 5 Key Laboratory of Glycoconjugate Research Ministry of Public Health, Department of Biochemistry and Molecular Biology, Shanghai Medical College, \\ Fudan University, Shanghai, China \\ ${ }^{6}$ Guangzhou Institute of Pediatrics, Guangzhou Women and Children's Medical Center, Guangzhou Medical University, Guangzhou, Guangdong Province, \\ China
}

Corresponding Authors: Jun Wang, Ling Dong

Email address: jwang03@sibs.ac.cn, dong.ling@zs-hospital.sh.cn

Robust and accurate gene expression signature is essential to assist oncologists to determine which subset of patients at similar Tumor-Lymph Node-Metastasis (TNM) stage has high recurrence risk and could benefit from adjuvant therapies. Here we applied a twostep supervised machine-learning method and established a 12-gene expression signature to precisely predict colon adenocarcinoma (COAD) prognosis by using COAD RNA-seq transcriptome data from The Cancer Genome Atlas (TCGA). The predictive performance of the 12-gene signature was validated with two independent gene expression microarray datasets: GSE39582 includes 566 COAD cases for the development of six molecular subtypes with distinct clinical, molecular and survival characteristics; GSE17538 is a dataset containing 232 colon cancer patients for the generation of a metastasis gene expression profile to predict recurrence and death in COAD patients. The signature could effectively separate the poor prognosis patients from good prognosis group [disease specific survival (DSS): Kaplan Meier (KM) Log Rank $p=0.0034$; overall survival (OS): KM Log Rank $p=0.0336$ ] in GSE17538. For patients with proficient mismatch repair system (pMMR) in GSE39582, the signature could also effectively distinguish high risk group from low risk group [OS: KM Log Rank $p=0.005$; Relapse free survival (RFS): KM Log Rank $p=0.022$ ]. Interestingly, advanced stage patients were significantly enriched in high 12 gene score group (Fisher's exact test $p=0.0003$ ). After stage stratification, the signature could still distinguish poor prognosis patients in GSE17538 from good prognosis within stage II (Log Rank $p=0.01$ ) and stage II\&III (Log Rank $p=0.017)$ in the outcome of DFS. Within stage III or II / III pMMR patients treated with Adjuvant ChemoTherapies (ACT), 
patients with higher 12-gene score showed poorer prognosis (III, OS: KM Log Rank $p=0.046$; IIIII, OS: KM Log Rank $p=0.041$ ). Among stage II/III pMMR patients with lower 12-gene scores in GSE39582, subgroup receiving ACT showed significantly longer OS time compared with those who received no ACT (Log Rank $p=0.021$ ), while there is no obvious difference between counterparts among patients with higher 12-gene scores (Log Rank $p=0.12$ ). Besides COAD, our 12-gene signature is multifunctional in several other cancer types including kidney cancer, lung cancer, uveal and skin melanoma, brain cancer, and pancreatic cancer. Functional classification showed that the seven of the twelve genes are involved in immune system function and regulation. So our 12-gene signature could potentially be used to guide decisions about adjuvant therapy for patients with stage II/III and PMMR COAD. 


\section{Establishment of a 12-gene expression signature to predict colon cancer}

\section{prognosis}

3 Dalong Sun ${ }^{1 \#}$, Jing Chen ${ }^{2 \#}$, Longzi Liu ${ }^{3 \#}$, Guangxi Zhao ${ }^{4}$, Pingping Dong ${ }^{1}$, Bingrui Wu ${ }^{5}$, Jun 4 Wang $^{6 *}$, Ling Dong ${ }^{1 *}$

51 Department of Gastroenterology and Hepatology, Zhongshan Hospital, Fudan University, 6 Shanghai 200032, China;

72 Department of Neurology, Shanghai Fifth People's Hospital, Fudan University, Shanghai

8 200240, China;

93 Department of Hepatic Surgery, Liver Cancer Institute, and Key Laboratory of Carcinogenesis 10 and Cancer Invasion (Ministry of Education), Zhongshan Hospital, Fudan University, Shanghai 11 200032, China;

124 Department of Gastroenterology, Shanghai East Hospital, Tongji University School of 13 Medicine, Shanghai 200120, China;

145 Key Laboratory of Glycoconjugate Research Ministry of Public Health, Department of 15 Biochemistry and Molecular Biology, Shanghai Medical College, Fudan University, Shanghai 16 200032, China;

176 Guangzhou Institute of Pediatrics, Guangzhou Women and Children's Medical Center, 18 Guangzhou Medical University, Guangzhou, Guangdong, 510623, China;

19 \# These authors contributed equally.

20 Corresponding authors:

21 Ling Dong,

22 dong.ling@zs-hospital.sh.cn

23 Jun Wang,

24 jwang03@sibs.ac.cn 


\section{Abstract}

Robust and accurate gene expression signature is essential to assist oncologists to determine which subset of patients at similar Tumor-Lymph Node-Metastasis (TNM) stage has high recurrence risk and could benefit from adjuvant therapies. Here we applied a two-step supervised machine-learning method and established a 12-gene expression signature to precisely predict colon adenocarcinoma (COAD) prognosis by using COAD RNA-seq transcriptome data from The Cancer Genome Atlas (TCGA). The predictive performance of the 12-gene signature was validated with two independent gene expression microarray datasets: GSE39582 includes 566 COAD cases for the development of six molecular subtypes with distinct clinical, molecular and survival characteristics; GSE17538 is a dataset containing 232 colon cancer patients for the generation of a metastasis gene expression profile to predict recurrence and death in COAD patients. The signature could effectively separate the poor prognosis patients from good prognosis group [disease specific survival (DSS): Kaplan Meier (KM) Log Rank $p=0.0034$; overall survival (OS): KM Log Rank $p=0.0336]$ in GSE17538. For patients with proficient mismatch repair system (pMMR) in GSE39582, the signature could also effectively distinguish high risk group from low risk group [OS: KM Log Rank $p=0.005$; Relapse free survival (RFS): $\mathrm{KM} \log$ Rank $p=0.022]$. Interestingly, advanced stage patients were significantly enriched in high 12-gene score group (Fisher's exact test $p=0.0003$ ). After stage stratification, the signature could still distinguish poor prognosis patients in GSE17538 from good prognosis within stage II (Log Rank $p=0.01)$ and stage II\&III (Log Rank $p=0.017)$ in the outcome of DFS. Within stage III or II/III pMMR patients treated with Adjuvant ChemoTherapies (ACT), patients with higher 12-gene score showed poorer prognosis (III, OS: KM Log Rank $p=0.046$; III\&II, OS: KM Log Rank $p=0.041$ ). Among stage II/III pMMR patients with lower 12-gene scores in GSE39582, subgroup receiving ACT showed significantly longer OS time compared with those who received no ACT ( $\log \operatorname{Rank} p=0.021)$, while there is no obvious difference between counterparts among patients with higher 12-gene scores (Log Rank $p=0.12$ ). Besides COAD, our 12-gene signature is multifunctional in several other cancer types including kidney cancer, lung cancer, uveal and skin melanoma, brain cancer, and pancreatic cancer. Functional classification showed that the seven of the twelve genes are involved in immune system function and regulation. So our 12-gene signature could potentially be used to guide decisions about adjuvant therapy for patients with stage II/III and pMMR COAD. 


\section{Introduction}

Colorectal cancer (CRC) is one of the most common cancers in men and women, representing almost $10 \%$ of the global cancer incidents and the third leading cause of cancer death worldwide(McGuire, 2016). CRC comprises three different subtypes according to distinct pathway operate: chromosomal-instable, microsatellite-instable, and $\mathrm{CpG}$ island methylator phenotype, all of which differ in morphology, genetic background, molecular profile, clinical behavior, and response to therapy(De Sousa et al., 2013). Current prognostic model based on the classic tumor-node-metastasis (TNM) staging is the standard prognosis factor for CRC in clinical practice. However, due to the high heterogeneity of disease, the patients at similar stage behave differently in terms of recurrence and response to chemotherapy often differs. Better parameters to guide patients' prognostic stratification and personalized medicine are urgently needed. Currently, some prognostic and predictive molecular markers have been developed. Microsatellite instability (MSI) is the molecular hallmark of DNA mismatch repair (MMR) deficiency. In stage II disease, MSI status helps select patients with high risk of developing recurrence(Brychtova et al., 2017). MSI status can also be a predictor of the benefit of adjuvant chemotherapy with fluorouracil in stage II and stage III colon cancer(Ribic et al., 2003). KRAS mutation status has been validated as a molecular marker for prediction of non-response to EGFR targeted drugs in metastatic CRC (Cunningham et al., 2010; Karapetis et al., 2008; Siena et al., 2009). However, due to complex pathways contributing to cancer progression, single molecular marker might not be efficient enough to predict prognosis and individualize in selecting adjuvant therapy.

The development of gene expression profiling technologies such as microarray and Next Generation Sequencing (NGS) provide further opportunities to comprehensively characterize the molecular features of cancer. Gene-expression profiling has been used to develop genomic tests that may provide better predictions of clinical outcomes in combination with traditional clinicopathologic factors (Gray et al., 2007; Venook et al., 2011; Meropol et al., 2011; Ebata et al., 2016; Moloney \& Cotter, 2017; Guinney et al., 2015; Marisa et al., 2013; Smith et al., 2010; Gentles et al., 2015). Some commercially genomic assays are available for the prediction of clinical outcome in CRC patients. The most well-known one is the Oncotype DX Colon Cancer Assay, which is a 12-gene ( 7 cancer related genes and 5 reference genes) genomic test that has been used to help identify individuals with high recurrence risk from stage II colon cancer patients with T3 and MMR proficient tumors (Gray et al., 2007; Venook et al., 2011; Meropol et al., 2011). However, the five reference genes in Oncotype DX Assay contain PGK1 and GPX1, which are important players in the process of energy metabolism and cellular oxidative stress, both of which are actively involved in cancer development and metastasis(Ebata et al., 2016; Moloney \& Cotter, 2017). Normalization with PGK1 and GPX1 might have diluted the tumorous heterogeneities among cancer patients. In this work, we applied two steps of supervised machine-learning method and established a 12-gene expression signature to precisely predict colon adenocarcinoma (COAD) prognosis by exhaustively using expression of all genes of TCGA COAD patients. 
100

101

102

103

104

105

106

107

108

109

110

111

112

113

114

115

116

117

118

119

120

121

122

123

124

125

126

127

128

129

130

131

132

133

134

\section{Materials and Methods}

\section{TCGA and GEO datasets}

RNA-seq data and clinic information for all cancer types were obtained from the Cancer Genome Altas (TCGA) RNA-seq database (https://cancergenome.nih.gov/). Microarray expression data and clinic information for COAD patients were retrieved from Gene Expression Omnibus (GEO) database (https://www.ncbi.nlm.nih.gov/geo/).

\section{Development of the gene expression signature}

The development process has training and validation phase.

\section{Training stage has two phases:}

\section{Phase I}

\section{Grouping}

The TCGA colon adenocarcinoma (COAD) patients were used for the development of prototype of the 118-gene signature that could predict COAD prognosis. We applied a similar supervised machine learning method that was used for MammaPrint(van T et al., 2002). Fortytwo patients experienced relapse within 3 years were designated as poor prognosis. Forty-nine patients who were relapse free for at least three years were categorized as good prognosis. The gene expression values were centered and scaled before grouping. For training dataset, 32 and 39 patients were randomly chosen from poor and good prognosis category, respectively. The rest patients were grouped as test dataset. Detailed clinic information was listed in Suppl. Table 1.

\section{Selection of genes with high correlation to real prognosis status}

Overall, there are 20530 genes in the raw RNA-seq data. The Pearson correlation coefficients with real prognosis status were calculated for all genes. Genes with absolute correlation coefficient greater than 0.3 were selected. To test whether such correlation coefficient distribution could be found by chance, a permutation method was used to generate 10,000 Monte-Carlo simulations randomizing the correlation between gene expression data of the 71 training patients and corresponding prognostic categories.

\section{Supervised machine-learning method}

Gene number incorporated in the signature needs to be optimized. 1510 genes were reordered by absolute coefficients from maximum to minimum. Starting from the top 2 genes on the list, 755 signatures were generated by adding two more genes from the top list each time until all the 1510 genes were exhaustively used as reporters. A Leave-One-Out Cross-Validation (LOOCV) method was employed to check the performances of these signatures:

Step 1: leave one tumor out;

Step 2: calculate the good- and poor-prognosis expression template by averaging the expression values for each gene incorporated in good-prognosis group and poor-prognosis group, 
135

136

137

138

139

140

141

142

143

144

145

146

147

148

149

150

151

152

153

154

155

156

157

158

159

160

161

162

163

164

165

166

167

168

169

170

171

172

173

174

respectively. Then we defined a parameter called risk coefficient (risk-coef.). For a tumor, risk coefficient was calculated with its gene expression profile and good- and poor-prognosis expression template:

Risk-coef $=$ cor-coef. to good template - cor-coef. to poor template;

Step 3, calculate the risk-coefs for all the remaining 70 training samples and the left out sample. Reorder the 71 samples by ranking their risk-coefs from small to large. Determine the genomic risk by taking first 32 tumors as high genomic risk and the rest 39 as low genomic risk. Check the consistency between genomic risk and real risk for the left out sample;

Step 4, repeat step 1-3 iteratively until all the 71 samples have been left out once. Collect the error counts when there is a disagreement between genomic risk and real risk for the left out sample.

Better signatures with least error counts were selected.

\section{Cross-validation without information leak}

The 1510 genes were obtained using all training samples including the one left out for cross validation. So there might have over-fitting issue due to information leak. A modified LOOCV with no information leak was performed as below:

Step 1, leave one patient out;

Step 2, calculate the Pearson correlation coefficients with real prognosis status for all genes with the reminding 70 training samples. Filter the genes with $\mid$ coefficient $\mid>=0.3$.

Step 3, generate the signature with the genes selected and predict the genomic risk for the left out sample.

Step 4, repeat step 1-3 iteratively until all the 71 samples have been left out once.

\section{Phase II}

Further machine learning process was applied to generate a concise scoring system. Before machine learning, the RPKM (Reads Per Kilobase per Million mapped reads) values need normalization, which was done through dividing them by geometric mean of RPKM values of TFRC, GUSB, and RPLP0. Firstly, the TCGA COAD patients (Suppl. Table 2) were split into training and test dataset. There is no significant difference between the clinicopathologic factors of these two groups (Table 1). For each of the 118 genes, we calculated the coefficient and $p$ value in univariate Cox Proportional Hazard regression model $(\mathrm{CPH})$ with training dataset. Then we reordered the gene list by sorting the univaraite Cox-regression $p$-value from minimum to maximum. So the top genes have stronger correlations with cancer prognosis. Starting from the top one gene in the list, we added one more gene iteratively from the top for multivariate $\mathrm{CPH}$ analysis. In every iteration step, the fitness of established signature on test dataset was checked by calculating Kaplan Meier Log Rank $p$-value (KM-p). At the end of iteration, signature 
175

176

177

178

179

180

181

182

183

184

185

186

187

188

189

190

191

192

193

194

195

196

197

198

199

200

201

202

203

204

205

206

207

208

209

210

211

212

incorporating the top 12 genes has the minimum test dataset KM- $p$ and was deemed as the optimal one. The multivariate Cox coefficient of each gene in the final signature was extracted to generate the scoring system:

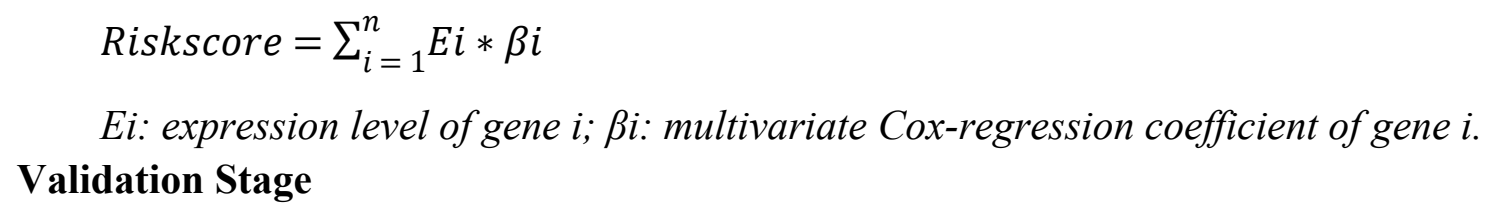

The GEO microarray datasets were used to validate the final gene expression signature. For genes with more than one probe, the probe showing minimum univariate $\mathrm{CPH} p$-value was selected. Relative expression level was obtained via dividing the probe signal by geometric mean of signals of TFRC, GUSB, and RPLP0. For each tumor, a risk score was obtained by calculating the weighted summation of relative expressions of the 12-gene. For a certain dataset, patients with risk scores below the median value of the population were designated as low risk group, while the rest of the patients were categorized as high risk group. Survival comparisons between high and low risk group were conducted by Kaplan-Meier plotting. Log Rank $p$ value $<0.05$ was considered as significantly different. Other cancer types in TCGA library were also retrieved to validate the 12 -gene signature.

\section{Results}

\section{Development of signature prototype}

The development process was shown as the flow chart in Figure 1. With the TCGA COAD data, an unbiased screening method was used to obtain 1510 genes showing absolute correlations greater than 0.3 with disease outcomes. The frequency distribution of number of genes with absolute coefficient no less than 0.3 in the 10,000 Monte-Carlo trials was displayed in Figure S1. The probability of obtaining 1510 genes or more with an absolute correlation coefficients of at least 0.3 with prognosis categories purely by chance was $0.0019(\mathrm{p}<0.05)$, which was fair for us to reject the null hypothesis.

During the (Leave-One-Out Cross Validation) LOOCV process, 755 signatures were generated. Least violation times were observed when signature employed the top 16, 36, 40, 42, $44,46,48,50,56,58,60,62,64,66,68,70,72,74,76,78,80,82,84,86$, or 118 genes. We further found that the predictive accuracy rates were high towards the 71 training samples with the signature containing the top 118 genes (Figure 2). We had the luxury to further validate the established signatures using the remaining 20 independent samples in test dataset. For each signature, receiver operating characteristic curve (ROC) was plotted with the information of riskcoefs and real risk of the 91 TCGA patients to compare the performances of the 25 signatures. There was no significant difference among the performances of these signatures (Figure 3 and Table 2).

Because the above 1510 genes were obtained using all the training samples including the 
213 one left out for cross validation, a modified LOOCV without information leak was performed.

214 Seventy-one additional signatures were created. The vast majority of the original 1510 genes

215 were shared by most of the 71 signatures (Figure S2). So there was very limited information

216 leak introduced during the previous training process.

217

\section{Development of 12-gene signature}

219 For the purpose of concise and simplicity, we further established a 12-gene expression signature

220

221

222

223

224

225

226

227

228

229

230

231

232

233

234

235

236

237

238

239

240

241

242

243

244

245

246

247

248

249

250

251

252

based on the 118 genes obtained in phase I training stage. Expressional coefficients were assigned to respective genes. Each patient has a risk score by calculating the weighted summation of expression values of the 12 genes. The Kaplan-Meier (KM) survival analysis showed that among TCGA COAD patients, the high risk group displayed significantly poorer prognosis than low risk group regarding to disease free survival (DFS) (training dataset: KM Log Rank $p=0.0001$; test dataset: KM Log Rank $p=0.0005$ ) (Figure 4).

\section{Prognostic values of the 12-gene signature in other COAD datasets}

GSE17538 (GSE17536 and GSE17537) was used to validate the 12-gene expression signature. With both clinic information and microarray gene expression of 232 colon cancer patients, Smith and Freeman $(15,18)$ established a metastasis gene expression profile to predict recurrence and death in COAD patients. The 12-gene signature could effectively separate the poor prognosis patients from good prognosis group [Figure 5 (a)-(c), Disease specific survival (DSS): KM Log Rank $p=0.0034$; Overall survival (OS): KM Log Rank $p=0.0336$; Disease free survival (DFS): KM Log Rank $p=0.0004]$. After stage stratification, the signature could still distinguish poor prognosis patients from good within stage II [Figure 5(d), Log Rank $p=0.01]$ and stage II\&III [Figure 5(e): $\log$ Rank $\mathrm{p}=0.017]$ in terms of DFS.

GSE39582 is a dataset including 566 COAD cases and 19 non-tumoral colorectal mucosas. With this dataset, Marisa et al. developed gene expression classification of colon cancer defining six molecular subtypes with distinct clinical, molecular and survival characteristics (19). In patients with proficient mismatch repair system (pMMR), our 12-gene signature could effectively distinguish high risk group from low risk group [Figure 6 (a) and (b), Relapse free survival (RFS): KM Log Rank $p=0.022$; OS: KM Log Rank $p=0.005]$. No significant difference was found in $\mathrm{KM}$ analysis performed among dMMR patients. Further survival analysis was performed within stage III or II\&III and pMMR patients treated with Adjuvant Chemotherapies (ACT): patients with higher 12-gene score showed poorer prognosis [Figure 6 (c) and (d): III, OS: KM Log Rank $p=0.046$; III\&II, OS: KM Log Rank $p=0.041]$. Interestingly, among stage II\&III pMMR patients with lower 12-gene scores, subgroup receiving adjuvant chemotherapies showed significantly longer OS time compared with those who received no adjuvant chemotherapy [Figure 6 (e): $\log$ Rank $p=0.021$ ], while there is no obvious difference between counterparts among patients with higher 12-gene scores[Figure 6 (f): $\log \operatorname{Rank} p=0.12$ ].

Interestingly, advanced stage patients were significantly enriched in high 12-gene score group (Table 3). 
253

254

255

256

257

258

259

260

261

262

263

264

265

266

267

268

269

270

271

272

273

274

275

276

277

278

279

280

281

282

283

284

285

286

287

288

289

290

291

292

\section{Predictive performances of the 12-gene signature in other cancer types}

We also tested the performance of the signature in other cancer types. TCGA RNA-seq data and corresponding clinic information for 24 cancer types were retrieved for validation. Surprisingly, KM results showed that our signature successfully separated good prognosis patients from poor prognosis patients in several other cancer types including pan-kidney cohort (KIPAN) (Figure 7a,OS: KM Log Rank $p=6.815 \mathrm{e}-6$ ), kidney renal clear cell carcinoma (KIRC) (Figure 7b, DFS: KM Log Rank $p=0.0480$ ), kidney renal papillary cell carcinoma (KIRP) (Figure 7c, DFS:KM Log Rank $p=0.0027$; Figure 7d OS: Log Rank $p=0.0129$ ), lung squamous cell carcinoma (LUSC) (Figure 7e, DFS: Log Rank $p=0.0071$ ), and skin cutaneous melanoma (SKCM) (Figure 7f, DFS: Log Rank $p=0.01117$ ), brain lower grade glioma (LGG) (Figure 8a, OS: Log Rank $p=0.0031)$, uveal melanoma (UVM) (Figure 8b, OS: Log Rank $p=0.0054)$, glioblastoma (GBM) (Figure 8c, OS: Log Rank $p=0.0074$ ), cervical and endocervical cancers (CESC) (Figure 8d, OS: Log Rank $p=0.0090$ ), pancreatic adenocarcinoma (PAAD) (Figure 8e, OS: Log Rank $p=0.0127)$, stomach adenocarcinoma (STAD) (Figure 8f, OS: Log Rank $p=0.0456)$.

\section{Discussion}

Numerous attempts have been made to establish gene expression signatures for the purpose of precise prediction of colorectal cancer prognosis(Gray et al., 2007; Venook et al., 2011; Meropol et al., 2011; Ebata et al., 2016; Moloney \& Cotter, 2017; Guinney et al., 2015; Marisa et al., 2013; Smith et al., 2010; Gentles et al., 2015). A meta-analysis was done to assess the clinical value of several published prognosis gene expression signatures in colorectal cancer (Sanz-Pamplona et al., 2012). Although most of the published signatures showed significant statistical association with prognosis, their accuracy to classify independent tumor samples into high-risk and low-risk group is limited. So we need more robust and accurate gene expression signature that can predict prognosis cross independent COAD datasets. Here we established a gene expression signature by applying two steps of supervised machine-learning method. The predicative accuracy of our gene expression signature was proven by validation in two large independent gene expression microarray datasets (GSE39582, N=459; GSE17538, N=232). Decision making regarding adjuvant therapy has been a debate among professional clinical organizations over the past 20 years (Dotan \& Cohen, 2011; Meropol, 2011; Vachani C, 2013). Currently speaking, uncertainty presents in adjuvant chemotherapeutic effect among stage II COAD patients who are mismatch repair system proficient. The Scottish Intercollegiate Guidelines Network (SIGN), ASCO, and NCCN are following different guidelines regarding this issue (Gao et al., 2016). Resectable COAD patients with pMMR routinely receive 5-FU based postoperative adjuvant chemotherapy (POCT) which has been shown to provide a relatively small absolute benefit (Andre et al., 2009; Gill et al., 2004; Sargent et al., 2009; Gray et al., 2011; Alex et al., 2017), indicating many COAD patients might have been over-treated due to the lacking of an effective test to stratify the patients further. Our gene signature showed important prognostic value for stage II or/and III pMMR COAD patients. There validation results in 
293

294

295

296

297

298

299

300

301

302

303

304

305

306

307

308

309

310

311

312

313

314

315

316

317

318

319

320

321

322

323

324

325

326

327

328

329

330

331

332

GSE39582 indicate that lower 12-gene score patients have gained survival benefit from adjuvant chemotherapies, while high score patients treated with adjuvant chemotherapies didn't receive survival benefit. So our 12-gene signature could potentially be used to guide decisions about adjuvant therapy for patients with stage II\&III and pMMR colon cancer.

Seven of the proteins encoded by the 12 genes were related to immune system, they are TREML2, PADI4, NCKIPSD, PTPRN, PGLYRP1, C5orf53, and TREML3, indicating the essential roles of deregulated immune response in COAD progression and metastasis (Suppl. Table 3). TREML2, acting as the counter-receptor for B7-H3, promotes $\mathrm{T}$ cell responses(Hashiguchi et al., 2008). PADI4 protein catalyzes the conversion of arginine to citrulline residue. With specific high expression in blood lymphocytes(Asaga et al., 2001; Anzilotti et al., 2010), PADI4 is believed to be an active autoimmune player in synovial tissue of rheumatoid arthritis (Chang et al., 2005). It is reported that cell free circulation PADI4 mRNA level (together with cfDNA, PPBP, and haptoglobin) in peripheral blood of non-small cell lung cancer patients was significantly higher than that in healthy donors. So PADI4 may serve as a potential marker for NSCLC diagnosis (Ulivi et al., 2013). As a member of protein tyrosine phosphatase (PTP), PTPRN is an autoantigen in the sera of insulin-dependent diabetes mellitus (IDDM) patients, making it a promising therapeutic target of autoimmunity in IDDM (Rabin et al., 1994; Solimena et al., 1996). Hypermethylation in PTPRN was associated with longer progression-free survival in ovarian cancer (Bauerschlag et al., 2011). If that is the case, hypomethylation (upregulated mRNA expression level) in PTPRN may be associated with poor prognosis, which is consistent with our results. NCKIPSD is a protein containing SH3 and proline-rich domains. Reports have shown that NCKIPSD is involved in the maintenance of sarcomeres and assembly of myofibrils into sarcomeres(Lim et al., 2001). A very recent study reported that NCKIPSDdownregulation and increased $\alpha$-tublin acetylation promotes stiffness of tumor stroma, which in turn, may inhibit chemotherapeutic drug uptake and regulate tumor sensitivity to chemotherapy, resulting in high risk of breast cancer recurrence within 5 years (You et al., 2017). Consistently, our findings also showed decreased NCKIPSD expression is associated with high risk of colon cancer recurrence. PGLYRP1 is a member of peptidoglycan recognition proteins which are conserved innate immunity proteins, recognize bacterial peptidoglycan, and play a role in antibacterial immunity and inflammation(Dziarski \& Gupta, 2010). PGLYRP1 interacts with Hsp70 to induces cytotoxic activity in tumor cells via TNFR1 receptor (Yashin et al., 2015). C5orf53 is also called IgA inducing protein, which enhances IgA secretion from B-cells stimulated via CD40 (Endsley et al., 2009). TREML3 is a inhibitory receptor involved in antigen processing(Cella et al., 1997). Numerous studies have shown that cancer patients' prognosis and sensitivity to therapy are closely associated with infiltration and density of immunologic cells within primary tumors (Wels et al., 2008; McConnell \& Yang, 2009; McLean et al., 2011; Sethi \& Kang, 2011; Smith \& Kang, 2013). Of particular note, by applying a novel machine-learning method, called Cell-type Identification By Estimating

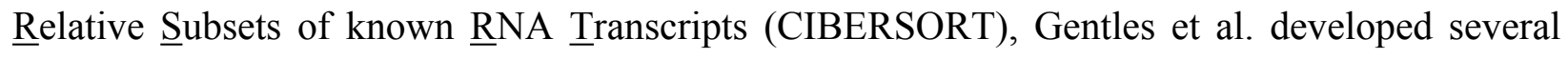
gene expression signatures to inferring distinct leukocyte subsets representation in bulk tumor 
333

334

335

336

337

338

339

340

341

342

343

344

345

346

347

348

349

350

351

352

353

354

355

356

357

358

359

360

361

362

363

364

365

366

367

368

369

370

371

transcriptomes (Gentles et al., 2015). In several solid tumors including colon cancer, the signatures relating to plasma cells and polymorphonuclear cells were the most significant favorable and adverse module to cancer outcomes, respectively. The broad spectrum involvement of lymphocyte infiltration and intra-tumor immune-suppression implies that this could be the main reason why our 12-gene signature could also predict patient prognosis in several other cancer types including kidney cancer, lung cancer, uveal and skin melanoma, brain cancer, and pancreatic cancer.

Other 5 genes (NOG, VIP, RIMKLB, NKAIN4, and FAM171B) in the 12-gene signature are functionally sporadic. NOG is related to mesodermal commitment and differentiation pathway(Costamagna et al., 2016). High expressing of gene signature including NOG showed a strong trend for a worse prognosis of patients with lung adenocarcinomas (Rajski et al., 2015). VIP, a member of glucagon/secretin superfamily, is the ligand of class II G protein-coupled receptor (Umetsu et al., 2011). It causes vasodilation and lowers arterial blood pressure. VIP signaling is enhanced in human prostate cancer (Fernandez-Martinez et al., 2012). Elevated VIP secretion is associated with advanced tumor stage in colorectal carcinoma (Hirayasu et al., 2002). RIMKLB is involved in alanine, aspartate and glutamate metabolism. RIMKLB upregulation is associated with radio-resistance in nasopharyngeal carcinomas ( $L i$ et al., 2016).NKAIN4 may interact with the beta subunit of Na, K-ATPase(Gorokhova et al., 2007). FAM171B which is a single-pass type I membrane protein, belongs to the FAM171 family. It is up-regulated in gemcitabine-resistant pancreatic cancer cell line (Zhou et al., 2015). The associations of these genes with cancer and cancer outcomes are very relevant to our findings in this study.

Our signature generated a novel scoring system with relative gene expression values by dividing the raw expression with geometric mean of RPKM values of three house-keeping genes (TFRC, GUSB, and RPLP0). In order to preserve the heterogeneities among tumors to the most extent, ACTB and GAPDH were avoided using as reference genes due to the fact that cytoskeleton and energy metabolism might be greatly deregulated among cancer individuals (Xiang et al., 2017; Stine \& Dang, 2013). A recent study overcomes hypoxia-induced tumor cell resistance by synergistic GAPDH-siRNA and chemotherapy (Guan et al., 2017), indicating the important roles of GAPDH in tumor cell resistance. Our normalization process also makes the gene expression scoring system very friendly to different gene expression detection systems including qPCR, RNA-seq, and QuantiGene Plex.

\section{Conclusion}

Robust and accurate gene expression signature is essential to assist oncologists to determine which subset of patients at similar TNM stage has high recurrence risk and could benefit from adjuvant therapies. Here we report a new 12-gene expression signature that could separate resectable COAD patients into poor- and good-prognosis group in several independent TCGA and microarray datasets. Functional classification showed that the seven of the twelve genes are 
372

373

374

375

376

377

378

379

380

381

382

383

384

385

386

387

388

389

390

391

392

393

394

395

396

397

398

399

400

401

402

403

404

405

406

407

408

409

410

involved in immune system function and regulation. Our gene expression signature could potentially serve as an effective genomic test in helping identify resectable COAD patients with high risk of poor prognosis. The accuracy and robustness of the signature as a prognostic classification requires further confirmation with large prospective patient cohorts.

\section{References:}

Alex, A.K., Siqueira, S., Coudry, R., Santos, J., Alves, M., Hoff, P.M., and Riechelmann, R.P. 2017. Response to Chemotherapy and Prognosis in Metastatic Colorectal Cancer With DNA Deficient Mismatch Repair. Clin Colorectal Cancer 16:228-239. DOI: 10.1016/j.clcc.2016.11.001

Andre, T., Boni, C., Navarro, M., Tabernero, J., Hickish, T., Topham, C., Bonetti, A., Clingan, P., Bridgewater, J., Rivera, F., and de Gramont, A. 2009. Improved overall survival with oxaliplatin, fluorouracil, and leucovorin as adjuvant treatment in stage II or III colon cancer in the MOSAIC trial. JOURNAL OF CLINICAL ONCOLOGY 27:3109-3116. DOI: 10.1200/JCO.2008.20.6771

Anzilotti, C., Pratesi, F., Tommasi, C., and Migliorini, P. 2010. Peptidylarginine deiminase 4 and citrullination in health and disease. AUTOIMMUNITY REVIEWS 9:158-160. DOI 10.1016/j.autrev.2009.06.002

Asaga, H., Nakashima, K., Senshu, T., Ishigami, A., and Yamada, M. 2001. Immunocytochemical localization of peptidylarginine deiminase in human eosinophils and neutrophils. J Leukoc Biol 70:46-51.

Bauerschlag, D.O., Ammerpohl, O., Brautigam, K., Schem, C., Lin, Q., Weigel, M.T., Hilpert, F., Arnold, N., Maass, N., Meinhold-Heerlein, I., and Wagner, W. 2011. Progression-free survival in ovarian cancer is reflected in epigenetic DNA methylation profiles. ONCOLOGY 80:12-20. DOI: 10.1159/000327746

Brychtova, V., Sefr, R., Hrstka, R., Videnska, P., Bencsikova, B., Hanakova, B., Zdrazilova, D.L., Nenutil, R., and Budinska, E. 2017. Molecular Pathology of Colorectal Cancer, Microsatellite Instability - the Detection, the Relationship to the Pathophysiology and Prognosis. Klin Onkol 30:153-155.

Cella, M., Dohring, C., Samaridis, J., Dessing, M., Brockhaus, M., Lanzavecchia, A., and Colonna, M. 1997. A novel inhibitory receptor (ILT3) expressed on monocytes, macrophages, and dendritic cells involved in antigen processing. JOURNAL OF EXPERIMENTAL MEDICINE 185:1743-1751.

Chang, X., Yamada, R., Suzuki, A., Sawada, T., Yoshino, S., Tokuhiro, S., and Yamamoto, K. 2005. Localization of peptidylarginine deiminase 4 (PADI4) and citrullinated protein in synovial tissue of rheumatoid arthritis. Rheumatology (Oxford) 44:40-50. DOI 10.1093/rheumatology/keh414Costamagna, D., Mommaerts, H., Sampaolesi, M., and Tylzanowski, P. 2016. Noggin inactivation affects the number and differentiation potential of muscle progenitor cells in vivo. Sci Rep 6:31949. DOI 10.1038/srep31949

Cunningham, D., Atkin, W., Lenz, H.J., Lynch, H.T., Minsky, B., Nordlinger, B., and Starling, N. 2010. Colorectal cancer. LANCET 375:1030-1047. DOI: 10.1016/S0140-6736(10)60353-4

De Sousa, E.M.F., Wang, X., Jansen, M., Fessler, E., Trinh, A., de Rooij, L.P., de Jong, J.H., de Boer, O.J., van Leersum, R., Bijlsma, M.F., Rodermond, H., van der Heijden, M., van Noesel, C.J., Tuynman, J.B., Dekker, E., Markowetz, F., Medema, J.P., and Vermeulen, L. 2013. Poor-prognosis colon cancer is defined by a molecularly distinct subtype and develops from serrated precursor lesions. NATURE MEDICINE 19:614618. DOI: $10.1038 / \mathrm{nm} .3174$

Dotan, E., and Cohen, S.J. 2011. Challenges in the management of stage II colon cancer. SEMINARS IN ONCOLOGY 38:511-520. DOI: 10.1053/j.seminoncol.2011.05.005 
Dziarski, R., and Gupta, D. 2010. Review: Mammalian peptidoglycan recognition proteins (PGRPs) in innate immunity. Innate Immun 16:168-174. DOI 10.1177/1753425910366059

Ebata, T., Hirata, H., and Kawauchi, K. 2016. Functions of the Tumor Suppressors p53 and Rb in Actin Cytoskeleton Remodeling. Biomed Research International 2016: 9231057. DOI: 10.1155/2016/9231057

Endsley, M.A., Njongmeta, L.M., Shell, E., Ryan, M.W., Indrikovs, A.J., Ulualp, S., Goldblum, R.M., Mwangi, W., and Estes, D.M. 2009. Human IgA-inducing protein from dendritic cells induces IgA production by naive IgD+ B cells. JOURNAL OF IMMUNOLOGY 182:1854-1859. DOI 10.4049/jimmunol.0801973

Fernandez-Martinez, A.B., Carmena, M.J., Arenas, M.I., Bajo, A.M., Prieto, J.C., and Sanchez-Chapado, M. 2012. Overexpression of vasoactive intestinal peptide receptors and cyclooxygenase- 2 in human prostate cancer. Analysis of potential prognostic relevance. HISTOLOGY AND HISTOPATHOLOGY 27:1093-1101. DOI: 10.14670/HH-27.1093

Gao, S., Tibiche, C., Zou, J., Zaman, N., Trifiro, M., O'Connor-McCourt, M., and Wang, E. 2016. Identification and Construction of Combinatory Cancer Hallmark-Based Gene Signature Sets to Predict Recurrence and Chemotherapy Benefit in Stage II Colorectal Cancer. JAMA Oncology 2:37-45. DOI: 10.1001/jamaoncol.2015.3413

Gentles, A.J., Newman, A.M., Liu, C.L., Bratman, S.V., Feng, W., Kim, D., Nair, V.S., Xu, Y., Khuong, A., Hoang, C.D., Diehn, M., West, R.B., Plevritis, S.K., and Alizadeh, A.A. 2015. The prognostic landscape of genes and infiltrating immune cells across human cancers. NATURE MEDICINE 21:938-945. DOI: 10.1038/nm.3909

Gill, S., Loprinzi, C.L., Sargent, D.J., Thome, S.D., Alberts, S.R., Haller, D.G., Benedetti, J., Francini, G., Shepherd, L.E., Francois, S.J., Labianca, R., Chen, W., Cha, S.S., Heldebrant, M.P., and Goldberg, R.M. 2004. Pooled analysis of fluorouracil-based adjuvant therapy for stage II and III colon cancer: who benefits and by how much? JOURNAL OF CLINICAL ONCOLOGY 22:1797-1806. DOI: 10.1200/JCO.2004.09.059

Gorokhova, S., Bibert, S., Geering, K., and Heintz, N. 2007. A novel family of transmembrane proteins interacting with beta subunits of the Na,K-ATPase. HUMAN MOLECULAR GENETICS 16:2394-2410. DOI 10.1093/hmg/ddm167

Gray, R., Barnwell, J., McConkey, C., Hills, R.K., Williams, N.S., and Kerr, D.J. 2007. Adjuvant chemotherapy versus observation in patients with colorectal cancer: a randomised study. LANCET 370:2020-2029. DOI: 10.1016/S0140-6736(07)61866-2

Gray, R.G., Quirke, P., Handley, K., Lopatin, M., Magill, L., Baehner, F.L., Beaumont, C., Clark-Langone, K.M., Yoshizawa, C.N., Lee, M., Watson, D., Shak, S., and Kerr, D.J. 2011. Validation study of a quantitative multigene reverse transcriptase-polymerase chain reaction assay for assessment of recurrence risk in patients with stage II colon cancer. JOURNAL OF CLINICAL ONCOLOGY 29:4611-4619. DOI: 10.1200/JCO.2010.32.8732

Guan, J., Sun, J., Sun, F., Lou, B., Zhang, D., Mashayekhi, V., Sadeghi, N., Storm, G., Mastrobattista, E., and He, Z. 2017. Hypoxia-induced tumor cell resistance is overcome by synergistic GAPDH-siRNA and chemotherapy co-delivered by long-circulating and cationic-interior liposomes. Nanoscale 9:9190-9201. DOI: $10.1039 / \mathrm{c} 7 \mathrm{nr} 02663 \mathrm{c}$

Guinney, J., Dienstmann, R., Wang, X., de Reynies, A., Schlicker, A., Soneson, C., Marisa, L., Roepman, P., Nyamundanda, G., Angelino, P., Bot, B.M., Morris, J.S., Simon, I.M., Gerster, S., Fessler, E., De Sousa, E.M.F., Missiaglia, E., Ramay, H., Barras, D., Homicsko, K., Maru, D., Manyam, G.C., Broom, B., Boige, 
V., Perez-Villamil, B., Laderas, T., Salazar, R., Gray, J.W., Hanahan, D., Tabernero, J., Bernards, R., Friend, S.H., Laurent-Puig, P., Medema, J.P., Sadanandam, A., Wessels, L., Delorenzi, M., Kopetz, S., Vermeulen, L., and Tejpar, S. 2015. The consensus molecular subtypes of colorectal cancer. NATURE MEDICINE 21:1350-1356. DOI: 10.1038/nm.3967

Hashiguchi, M., Kobori, H., Ritprajak, P., Kamimura, Y., Kozono, H., and Azuma, M. 2008. Triggering receptor expressed on myeloid cell-like transcript 2 (TLT-2) is a counter-receptor for B7-H3 and enhances T cell responses. Proc Natl Acad Sci U S A 105:10495-10500. DOI 10.1073/pnas.0802423105

Hirayasu, Y., Oya, M., Okuyama, T., Kiumi, F., and Ueda, Y. 2002. Vasoactive intestinal peptide and its relationship to tumor stage in colorectal carcinoma: an immunohistochemical study. JOURNAL OF GASTROENTEROLOGY 37:336-344. DOI: 10.1007/s005350200047

Karapetis, C.S., Khambata-Ford, S., Jonker, D.J., O'Callaghan, C.J., Tu, D., Tebbutt, N.C., Simes, R.J., Chalchal, H., Shapiro, J.D., Robitaille, S., Price, T.J., Shepherd, L., Au, H.J., Langer, C., Moore, M.J., and Zalcberg, J.R. 2008. K-ras mutations and benefit from cetuximab in advanced colorectal cancer. $N$ Engl $J$ Med 359:1757-1765. DOI: 10.1056/NEJMoa0804385

Li, G., Liu, Y., Liu, C., Su, Z., Ren, S., Wang, Y., Deng, T., Huang, D., Tian, Y., and Qiu, Y. 2016. Genomewide analyses of long noncoding RNA expression profiles correlated with radioresistance in nasopharyngeal carcinoma via next-generation deep sequencing. BMC CANCER 16:719. DOI: 10.1186/s12885-016-2755-6

Lim, C.S., Park, E.S., Kim, D.J., Song, Y.H., Eom, S.H., Chun, J.S., Kim, J.H., Kim, J.K., Park, D., and Song, W.K. 2001. SPIN90 (SH3 protein interacting with Nck, $90 \mathrm{kDa}$ ), an adaptor protein that is developmentally regulated during cardiac myocyte differentiation. JOURNAL OF BIOLOGICAL CHEMISTRY 276:1287112878. DOI 10.1074/jbc.M009411200

Marisa, L., de Reynies, A., Duval, A., Selves, J., Gaub, M.P., Vescovo, L., Etienne-Grimaldi, M.C., Schiappa, R., Guenot, D., Ayadi, M., Kirzin, S., Chazal, M., Flejou, J.F., Benchimol, D., Berger, A., Lagarde, A., Pencreach, E., Piard, F., Elias, D., Parc, Y., Olschwang, S., Milano, G., Laurent-Puig, P., and Boige, V. 2013. Gene expression classification of colon cancer into molecular subtypes: characterization, validation, and prognostic value. PLOS MEDICINE 10:e1001453. DOI: 10.1371/journal.pmed.1001453

McConnell, B.B., and Yang, V.W. 2009. The Role of Inflammation in the Pathogenesis of Colorectal Cancer. Curr Colorectal Cancer Rep 5:69-74. DOI: 10.1007/s11888-009-0011-z

McGuire, S. 2016. World Cancer Report 2014. Geneva, Switzerland: World Health Organization, International Agency for Research on Cancer, WHO Press, 2015. Advances in Nutrition 7:418-419. DOI: 10.3945/an.116.012211

McLean, M.H., Murray, G.I., Stewart, K.N., Norrie, G., Mayer, C., Hold, G.L., Thomson, J., Fyfe, N., Hope, M., Mowat, N.A., Drew, J.E., and El-Omar, E.M. 2011. The inflammatory microenvironment in colorectal neoplasia. PLoS One 6:e15366. DOI: 10.1371/journal.pone.0015366

Meropol, N.J. 2011. Ongoing challenge of stage II colon cancer. JOURNAL OF CLINICAL ONCOLOGY 29:33463348. DOI: $10.1200 / J C O .2011 .35 .4571$

Meropol, N.J., Lyman, G.H., Chien, R., and Hornberger, J.C. 2011. Use of a multigene prognostic assay for selection of adjuvant chemotherapy in patients with stage II colon cancer: Impact on quality-adjusted life expectancy and costs. JOURNAL OF CLINICAL ONCOLOGY 29S. DOI: 10.1200/jco.2011.29.4_suppl.491

Moloney, J.N., and Cotter, T.G. 2017. ROS signalling in the biology of cancer. SEMINARS IN CELL \& DEVELOPMENTAL BIOLOGY. DOI: 10.1016/j.semcdb.2017.05.023 
Rabin, D.U., Pleasic, S.M., Shapiro, J.A., Yoo-Warren, H., Oles, J., Hicks, J.M., Goldstein, D.E., and Rae, P.M. 1994. Islet cell antigen 512 is a diabetes-specific islet autoantigen related to protein tyrosine phosphatases. JOURNAL OF IMMUNOLOGY 152:3183-3188.

Rajski, M., Saaf, A., and Buess, M. 2015. BMP2 response pattern in human lung fibroblasts predicts outcome in lung adenocarcinomas. BMC Medical Genomics 8:16. DOI: 10.1186/s12920-015-0090-4

Ribic, C.M., Sargent, D.J., Moore, M.J., Thibodeau, S.N., French, A.J., Goldberg, R.M., Hamilton, S.R., Laurent-Puig, P., Gryfe, R., Shepherd, L.E., Tu, D., Redston, M., and Gallinger, S. 2003. Tumor microsatellite-instability status as a predictor of benefit from fluorouracil-based adjuvant chemotherapy for colon cancer. $N$ Engl J Med 349:247-257. DOI: 10.1056/NEJMoa022289

Sanz-Pamplona, R., Berenguer, A., Cordero, D., Riccadonna, S., Sole, X., Crous-Bou, M., Guino, E., Sanjuan, X., Biondo, S., Soriano, A., Jurman, G., Capella, G., Furlanello, C., and Moreno, V. 2012. Clinical value of prognosis gene expression signatures in colorectal cancer: a systematic review. PLoS One 7:e48877. DOI: 10.1371/journal.pone.0048877

Sargent, D., Sobrero, A., Grothey, A., O'Connell, M.J., Buyse, M., Andre, T., Zheng, Y., Green, E., Labianca, R., O'Callaghan, C., Seitz, J.F., Francini, G., Haller, D., Yothers, G., Goldberg, R., and de Gramont, A. 2009. Evidence for cure by adjuvant therapy in colon cancer: observations based on individual patient data from 20,898 patients on 18 randomized trials. JOURNAL OF CLINICAL ONCOLOGY 27:872-877. DOI: $10.1200 / \mathrm{JCO} .2008 .19 .5362$

Sethi, N., and Kang, Y. 2011. Unravelling the complexity of metastasis - molecular understanding and targeted therapies. NATURE REVIEWS CANCER 11:735-748. DOI: 10.1038/nrc3125

Siena, S., Sartore-Bianchi, A., Di Nicolantonio, F., Balfour, J., and Bardelli, A. 2009. Biomarkers predicting clinical outcome of epidermal growth factor receptor-targeted therapy in metastatic colorectal cancer. $J$ Natl Cancer Inst 101:1308-1324. DOI: 10.1093/jnci/djp280

Smith, H.A., and Kang, Y. 2013. The metastasis-promoting roles of tumor-associated immune cells. $J$ Mol Med (Berl) 91:411-429. DOI: 10.1007/s00109-013-1021-5

Smith, J.J., Deane, N.G., Wu, F., Merchant, N.B., Zhang, B., Jiang, A., Lu, P., Johnson, J.C., Schmidt, C., Bailey, C.E., Eschrich, S., Kis, C., Levy, S., Washington, M.K., Heslin, M.J., Coffey, R.J., Yeatman, T.J., Shyr, Y., and Beauchamp, R.D. 2010. Experimentally derived metastasis gene expression profile predicts recurrence and death in patients with colon cancer. GASTROENTEROLOGY 138:958-968. DOI: 10.1053/j.gastro.2009.11.005

Solimena, M., Dirkx, R.J., Hermel, J.M., Pleasic-Williams, S., Shapiro, J.A., Caron, L., and Rabin, D.U. 1996. ICA 512, an autoantigen of type I diabetes, is an intrinsic membrane protein of neurosecretory granules. EMBO JOURNAL 15:2102-2114.

Stine, Z.E., and Dang, C.V. 2013. Stress eating and tuning out: Cancer cells re-wire metabolism to counter stress. CRITICAL REVIEWS IN BIOCHEMISTRY AND MOLECULAR BIOLOGY 48:609-619. DOI: 10.3109/10409238.2013.844093

Ulivi, P., Mercatali, L., Casoni, G.L., Scarpi, E., Bucchi, L., Silvestrini, R., Sanna, S., Monteverde, M., Amadori, D., Poletti, V., and Zoli, W. 2013. Multiple marker detection in peripheral blood for NSCLC diagnosis. PLoS One 8:e57401. DOI: 10.1371/journal.pone.0057401

Umetsu, Y., Tenno, T., Goda, N., Shirakawa, M., Ikegami, T., and Hiroaki, H. 2011. Structural difference of vasoactive intestinal peptide in two distinct membrane-mimicking environments. Biochim Biophys Acta 
1814:724-730. DOI 10.1016/j.bbapap.2011.03.009

Vachani C, G.B. 2013. Stage II Colon Cancer: To Treat or Not to Treat? Available at https://www.oncolink.org/cancers/gastrointestinal/colon-cancer/treatments/stage-ii-colon-cancer-to-treat-ornot-to-treat (accessed April 18 2018).

van T, V.L., Dai, H., van de Vijver, M.J., He, Y.D., Hart, A.A., Mao, M., Peterse, H.L., van der Kooy, K., Marton, M.J., Witteveen, A.T., Schreiber, G.J., Kerkhoven, R.M., Roberts, C., Linsley, P.S., Bernards, R., and Friend, S.H. 2002. Gene expression profiling predicts clinical outcome of breast cancer. NATURE 415:530-536. DOI: $10.1038 / 415530 \mathrm{a}$

Venook, A.P., Niedzwiecki, D., Lopatin, M., Lee, M., Friedman, P.N., Frankel, W., Clark-Langone, K., Yoshizawa, C., Millward, C., Shak, S., Goldberg, R.M., Mahmoud, N.N., Schilsky, R.L., and Bertagnolli, M.M. 2011. Validation of a 12-gene colon cancer recurrence score (RS) in patients (pts) with stage II colon cancer (CC) from CALGB 9581. JOURNAL OF CLINICAL ONCOLOGY 29S:3518-3518.

Wels, J., Kaplan, R.N., Rafii, S., and Lyden, D. 2008. Migratory neighbors and distant invaders: tumor-associated niche cells. Genes Dev 22:559-574. DOI: 10.1101/gad.1636908

Xiang, C., Chen, J., and Fu, P. 2017. HGF/Met Signaling in Cancer Invasion: The Impact on Cytoskeleton Remodeling. Cancers 9:44. DOI: 10.3390/cancers9050044

Yashin, D.V., Ivanova, O.K., Soshnikova, N.V., Sheludchenkov, A.A., Romanova, E.A., Dukhanina, E.A., Tonevitsky, A.G., Gnuchev, N.V., Gabibov, A.G., Georgiev, G.P., and Sashchenko, L.P. 2015. Tag7 (PGLYRP1) in Complex with Hsp70 Induces Alternative Cytotoxic Processes in Tumor Cells via TNFR1 Receptor. JOURNAL OF BIOLOGICAL CHEMISTRY 290:21724-21731. DOI 10.1074/jbc.M115.639732

You, E., Huh, Y.H., Kwon, A., Kim, S.H., Chae, I.H., Lee, O.J., Ryu, J.H., Park, M.H., Kim, G.E., Lee, J.S., Lee, K.H., Lee, Y.S., Kim, J.W., Rhee, S., and Song, W.K. 2017. SPIN90 Depletion and Microtubule Acetylation Mediate Stromal Fibroblast Activation in Breast Cancer Progression. CANCER RESEARCH 77:4710-4722. DOI: 10.1158/0008-5472.CAN-17-0657

Zhou, M., Ye, Z., Gu, Y., Tian, B., Wu, B., and Li, J. 2015. Genomic analysis of drug resistant pancreatic cancer cell line by combining long non-coding RNA and mRNA expression profling. Int J Clin Exp Pathol 8:38-52. 


\section{Figure 1}

The flow chart of the development process of the COAD gene expression signature.

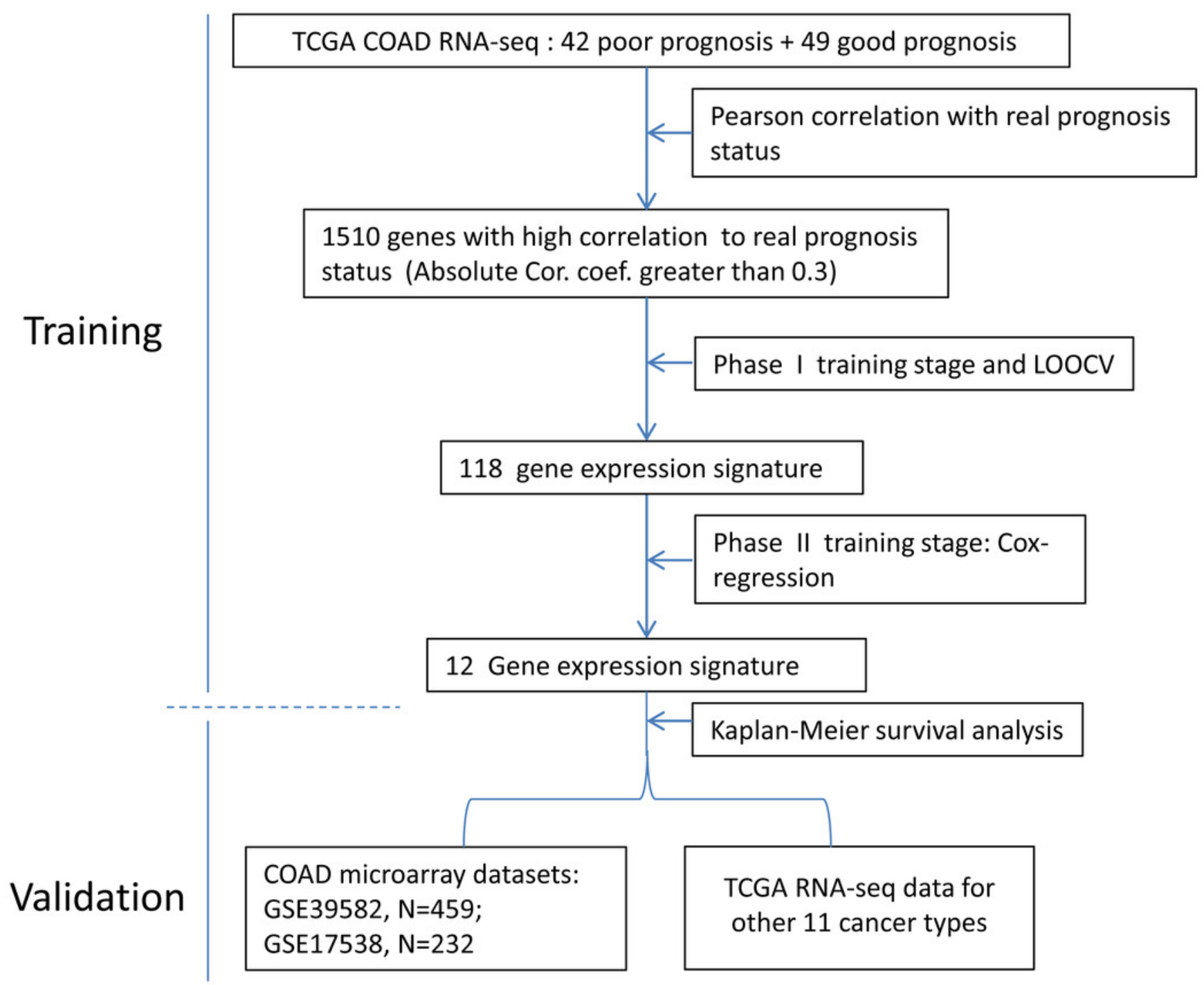




\section{Figure 2}

Prototype of the gene expression signature.

Expression heatmap plotting of 118 prognostic marker genes in training dataset (Up panel) and 20 patients in test dataset (Lower panel). Each row represents an observation (patient) and each column is a gene, whose name is labeled at the bottom. Tumors are ordered by the correlation to the average expression pattern of the good and poor prognosis group (Left panel). Genes are ordered by their correlation coefficients with the two prognosis categories. The real prognosis status for each tumor is displayed in the middle panel.

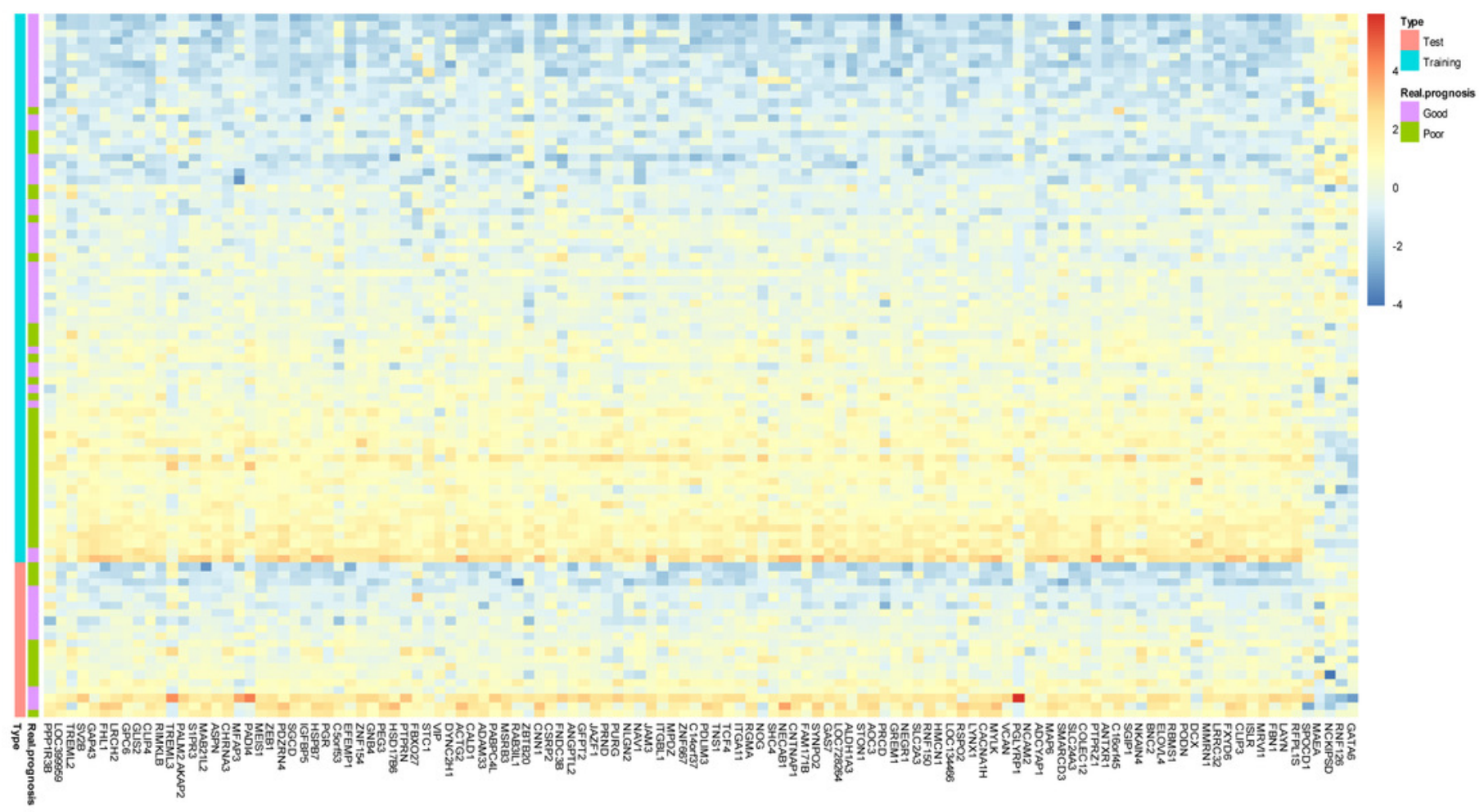


Figure 3

ROC plotting for the 25 signatures generated during phase I training process.

ROC with the information of risk-coefs and real risk of the 91 TCGA patients. ROC, receiver operating characteristic curve. TPR, true positive rate. FPR, false positive rate.

ROC

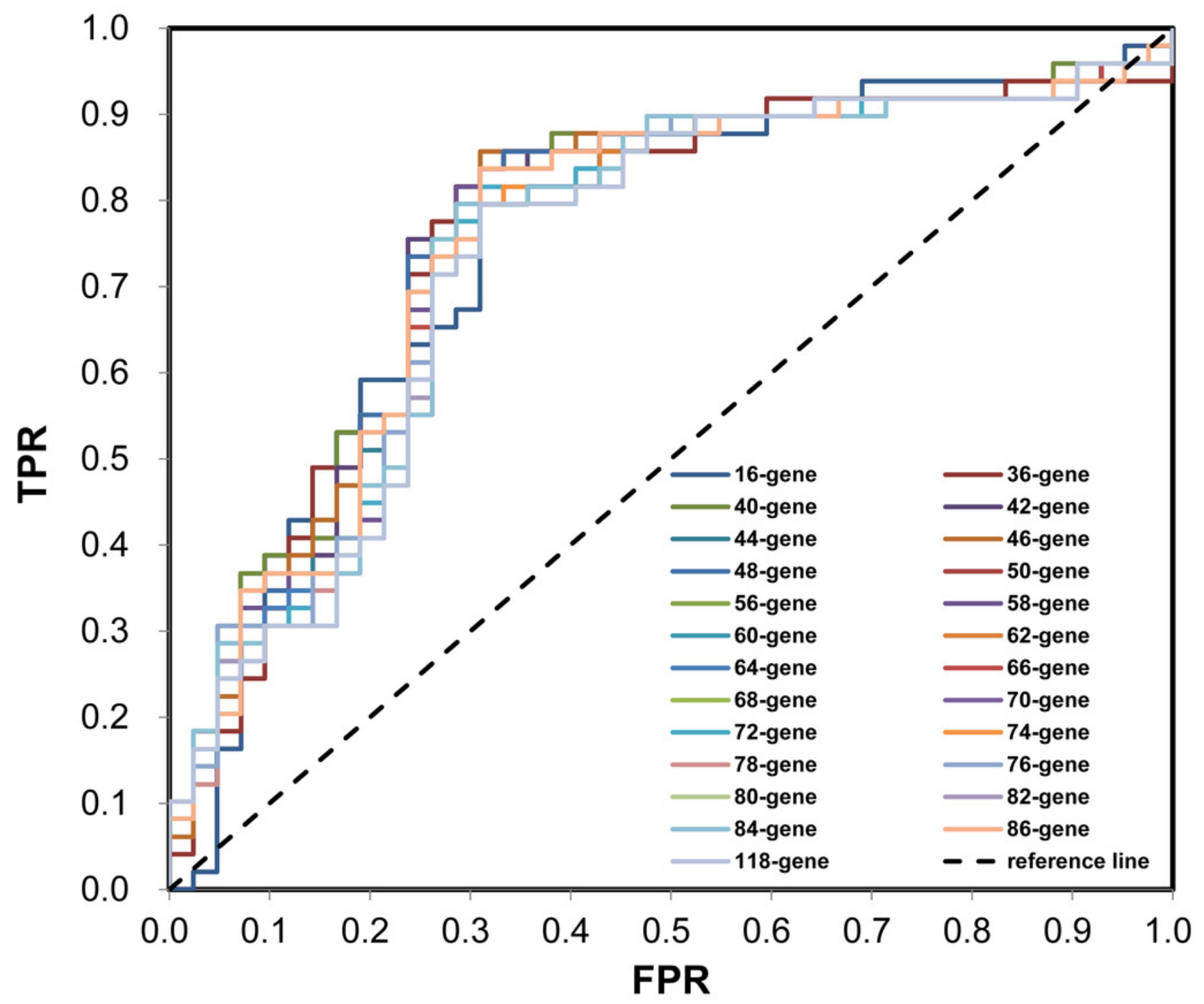


Figure 4

Prognostic values of the 12-gene signature.

Kaplan-Meier analysis of the high and low 12-gene risk score patients among TCGA COAD patients in training (a) and test dataset (b) in phase II training stage.

(a)

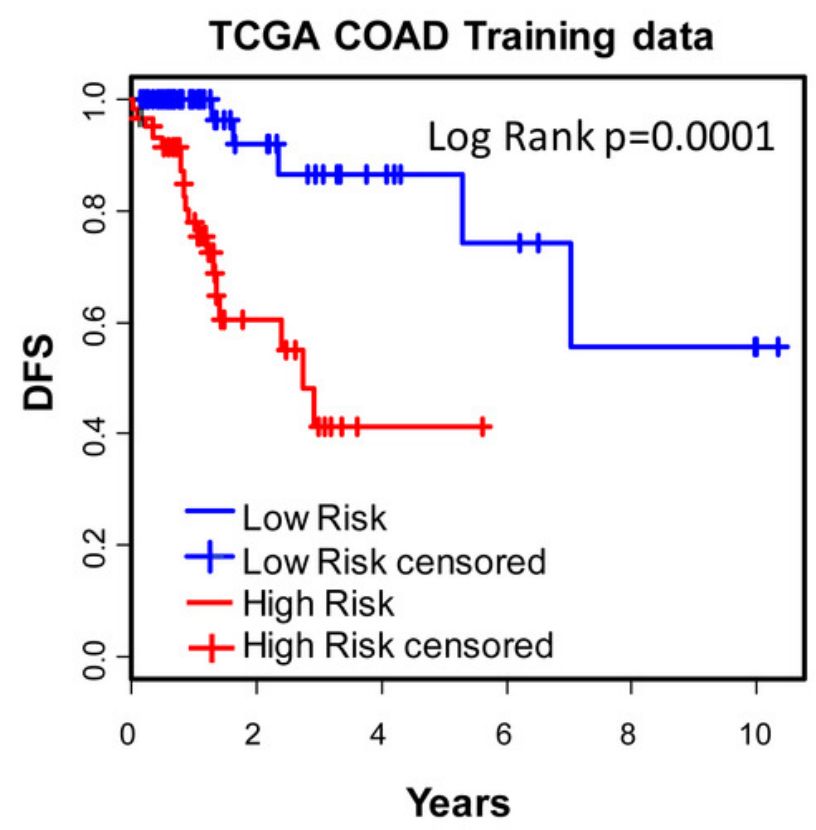

(b)

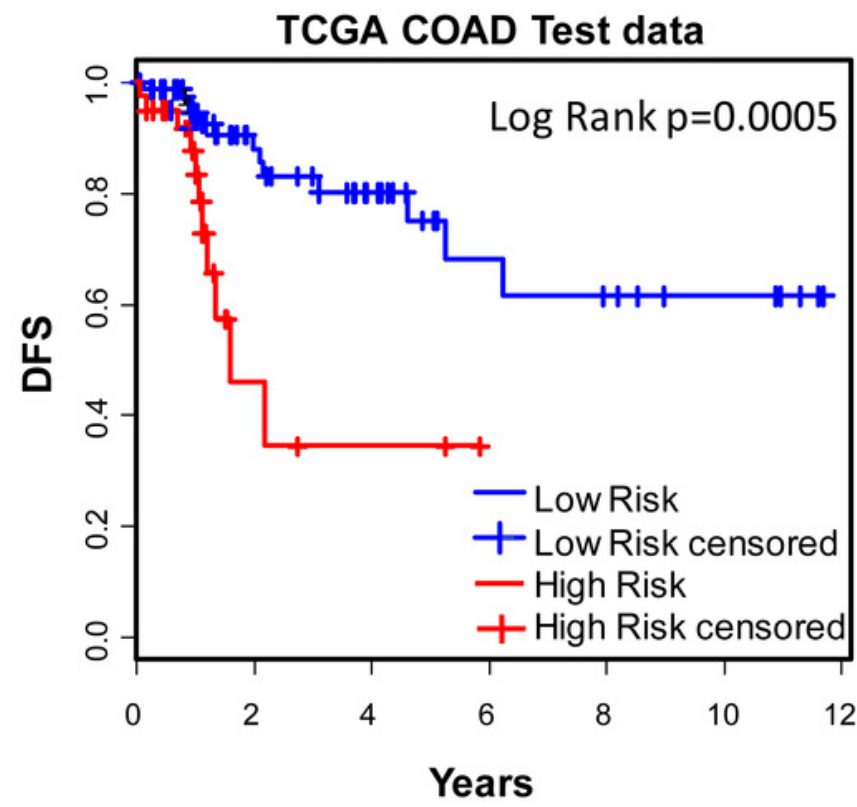




\section{Figure 5}

Prognostic values of the 12-gene signature in other COAD datasets.

(a)-(c): Kaplan-Meier curves showing patients (stagel-IV) with high and low12-gene risk score in endpoints of DSS, OS, and DFS, respectively; Kaplan-Meier curves showing patients at stage II (d) or II\&III (e) with high and low 12-gene risk score in terms of DFS. DFS, disease free survival; DSS, disease specific survival; OS, overall survival. 
(a)

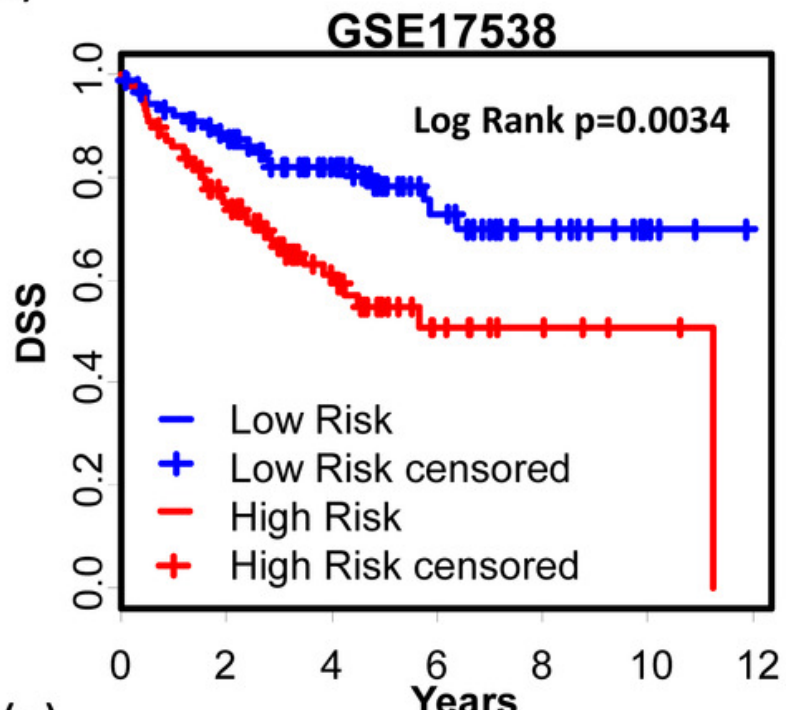

(c)

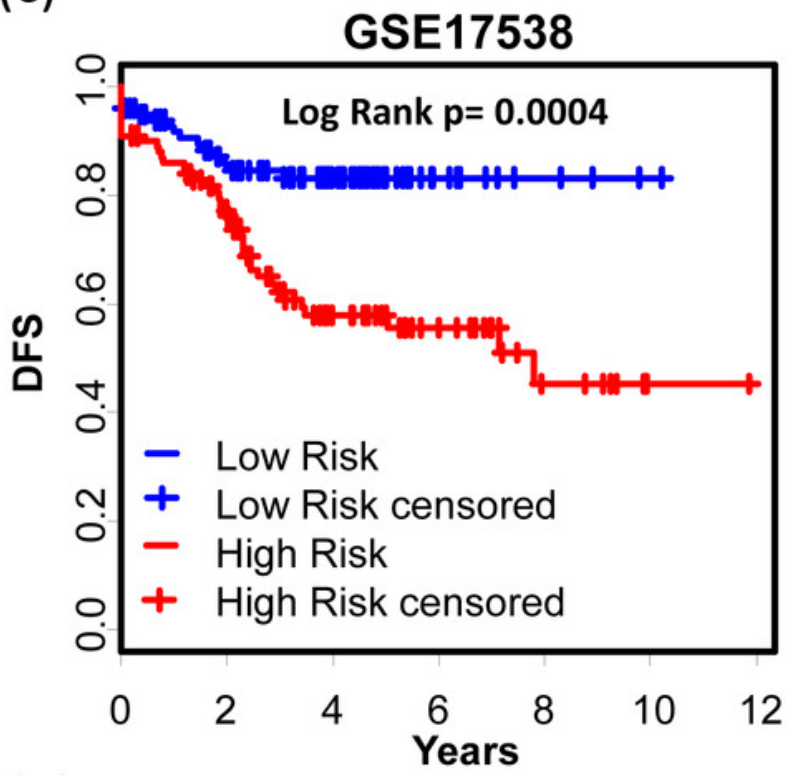

(e)

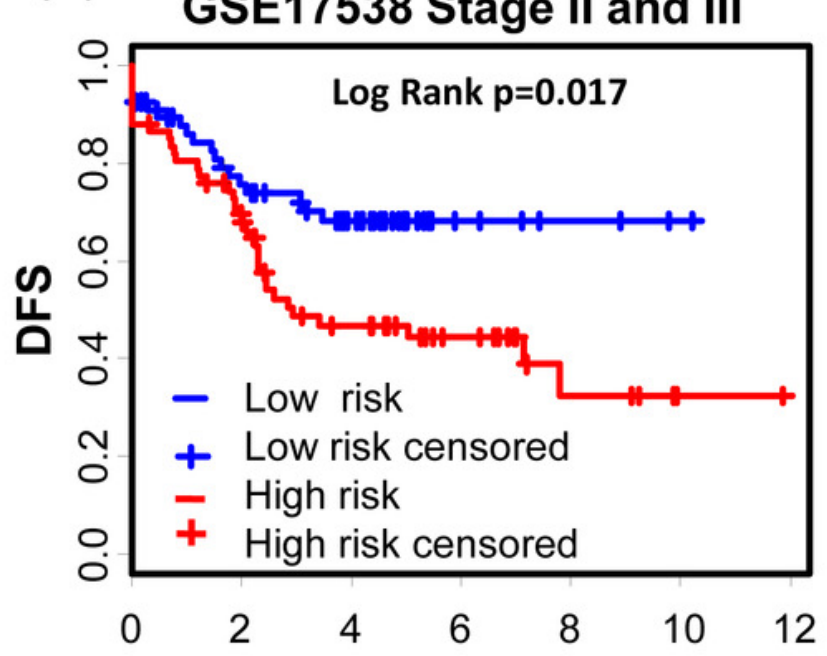

(b)

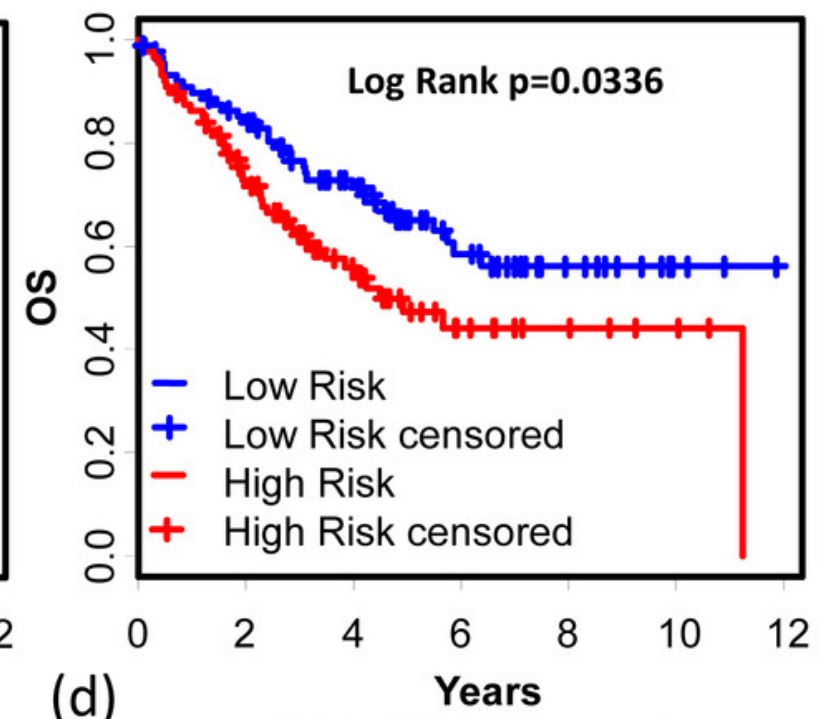

GSE17538 Stage II

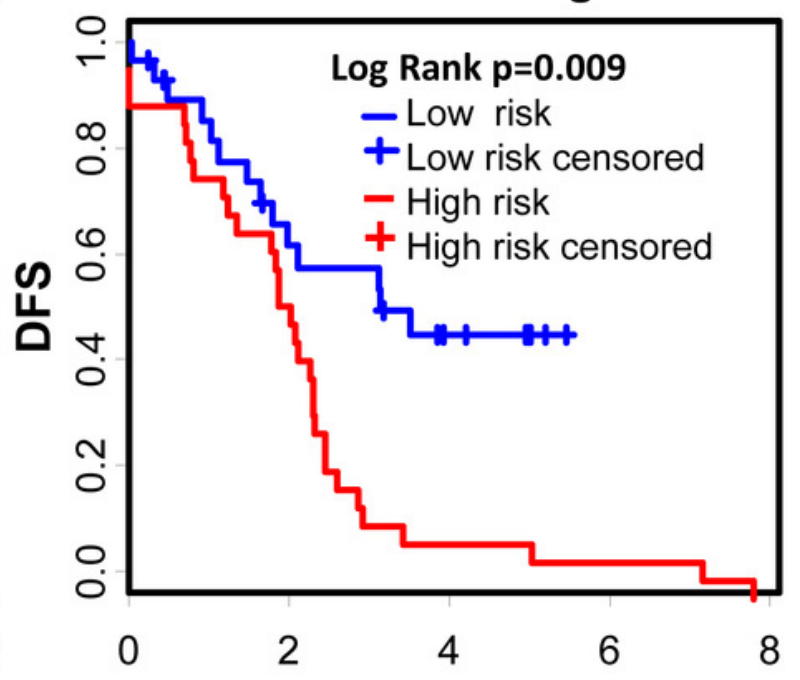




\section{Figure 6}

Prognostic values of the 12-gene signature in GSE39582.

(a) and (b): Kaplan-Meier curves showing patients (stagel-IV) with high and low 12-gene risk score in endpoints of RFS and OS, respectively; Kaplan-Meier curves showing stage III (c) or II\&III (d) pMMR patients (treated with ACT) with high and low 12-gene risk score in respect to the endpoint of OS; (e) In stage II\&III pMMR patients with low 12-gene scores, ACT subgroup displayed better OS outcome than control; (f) In stage II\&III pMMR patients with high 12-gene scores, ACT and control group displayed no significant difference in the outcome of OS. RFS: relapse-free survival; OS: overall survival; pMMR: proficient mismatch repair system. 
(a) GSE39582 pMMR

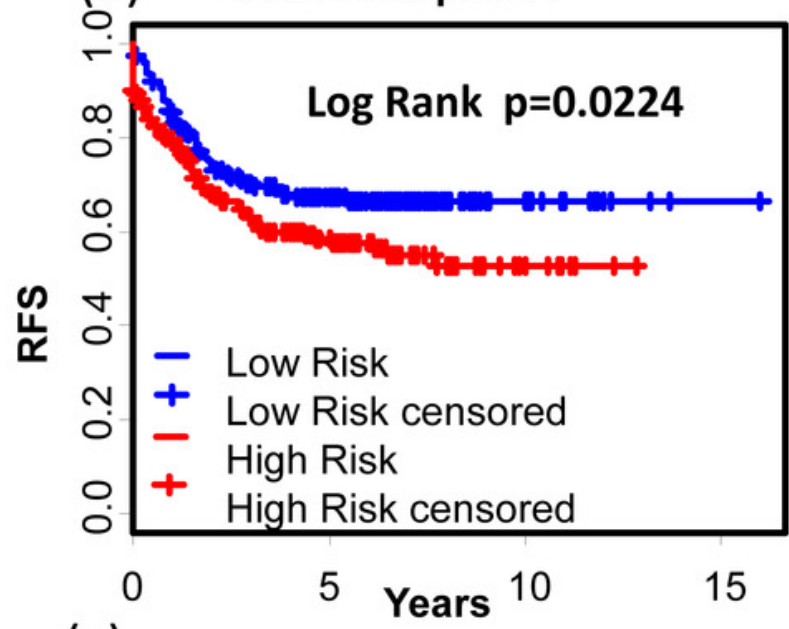

(c) GSE39582 Stage III pMMR patients with ACT

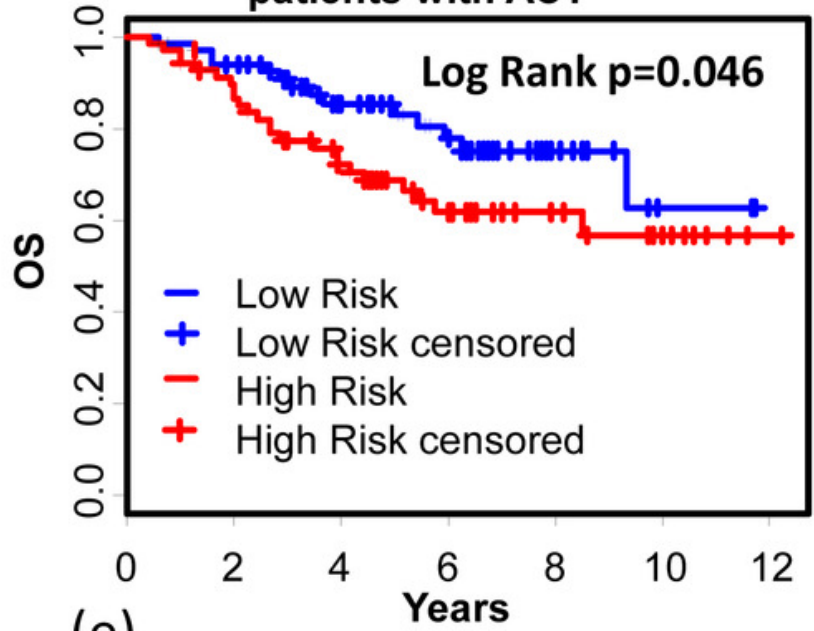

(e) GSE39582 Stage II\&III pMMR

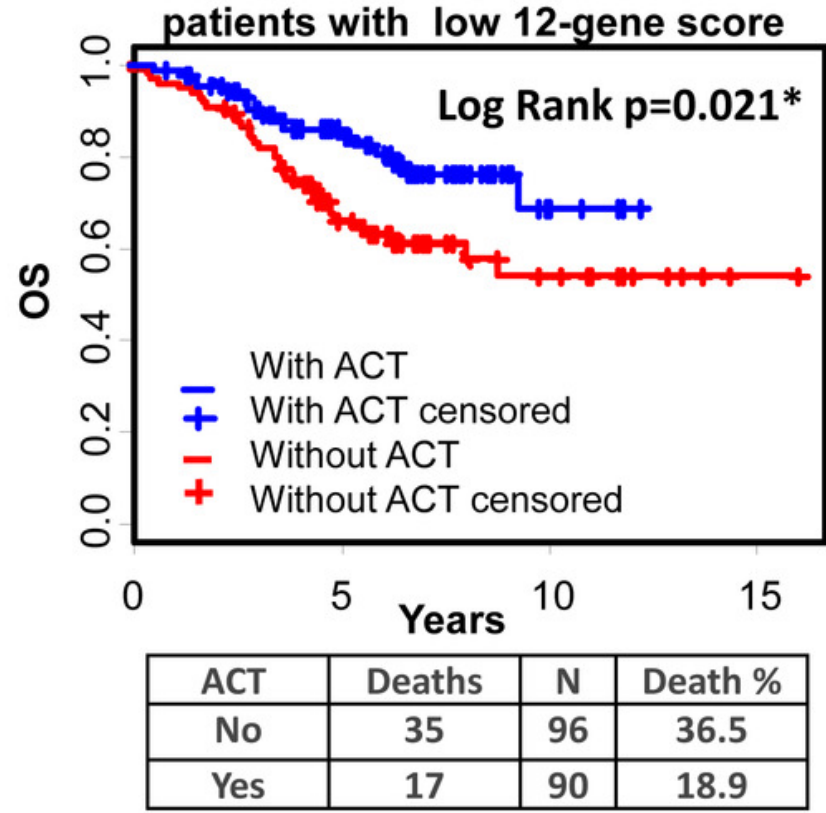

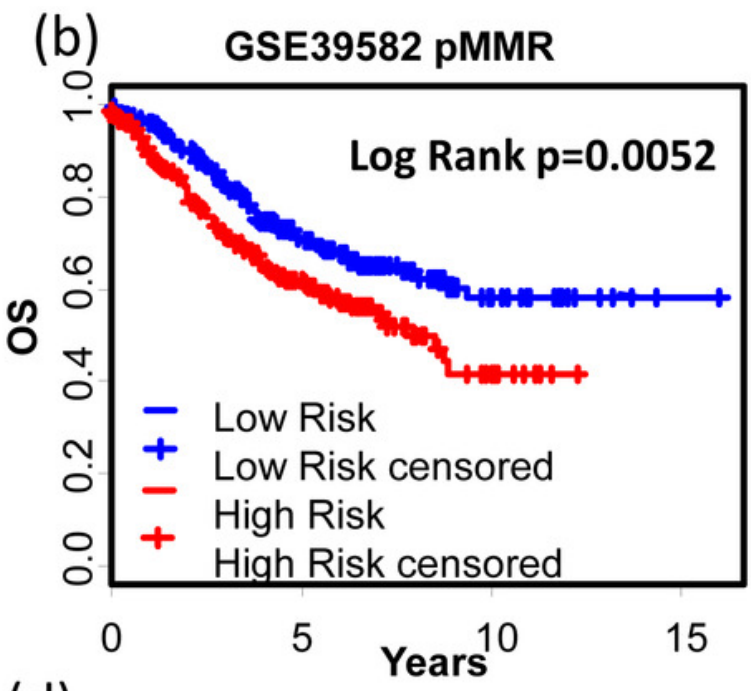

(d) GSE39582 Stage II\&III pMMR

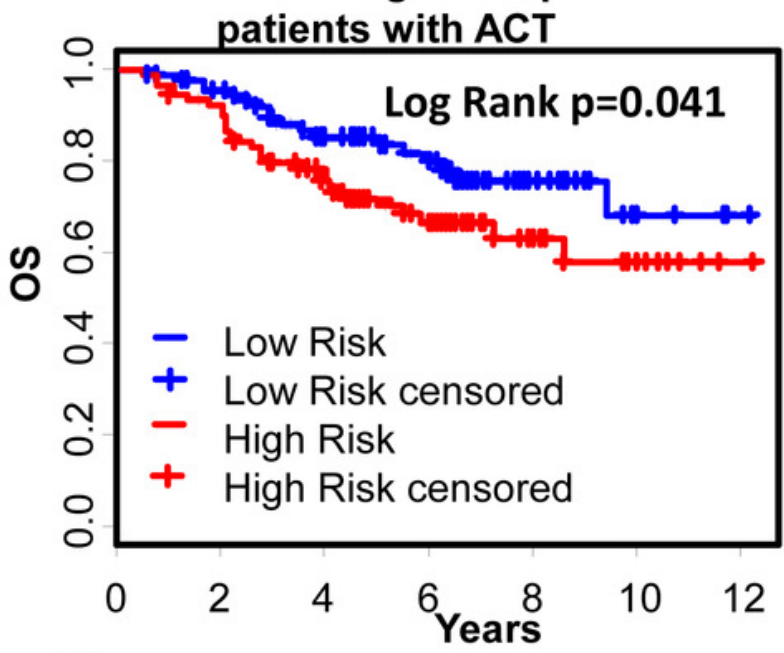

(f) GSE39582 Stage II \& III PMMR

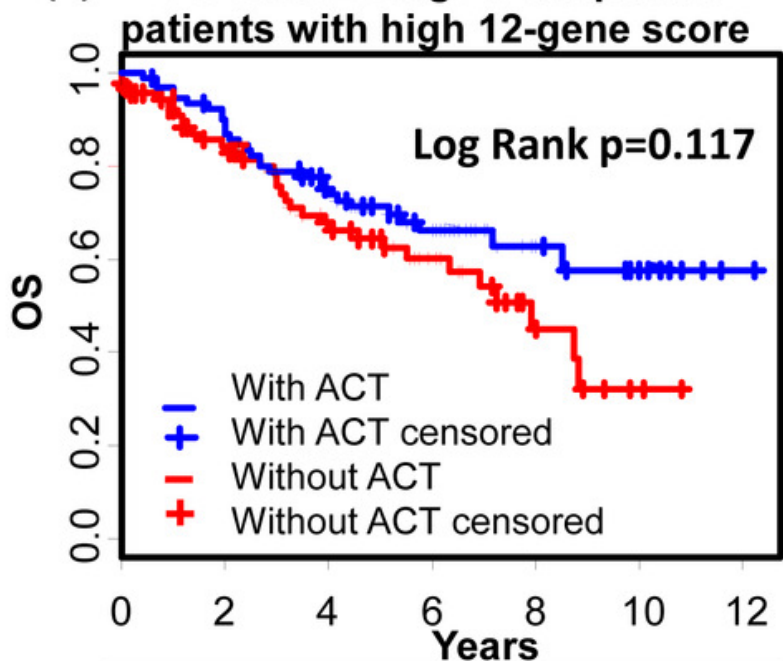

\begin{tabular}{|c|c|c|c|}
\hline ACT & Deaths & N & Death \% \\
\hline No & 34 & 92 & 37.0 \\
\hline Yes & 30 & 93 & 32.3 \\
\hline
\end{tabular}




\section{Figure 7}

KM analysis of the high and low 12-gene risk score patients for the major outcomes in other cancer types.

(a) OS in pan-kidney cohort (KIPAN), (b) DFS in kidney renal clear cell carcinoma (KIRC). (c) \& (d) DFS and OS in kidney renal papillary cell carcinoma (KIRP), respectively. (e) DFS in lung squamous cell carcinoma (LUSC). (f) DFS in skin cutaneous melanoma (SKCM). OS, overall survival. DFS, disease free survival. 
(a)

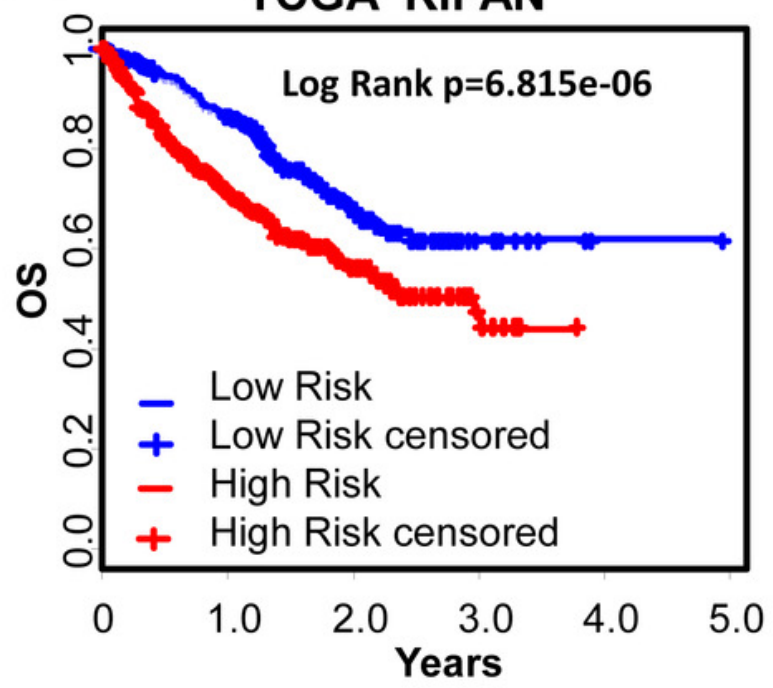

(c)
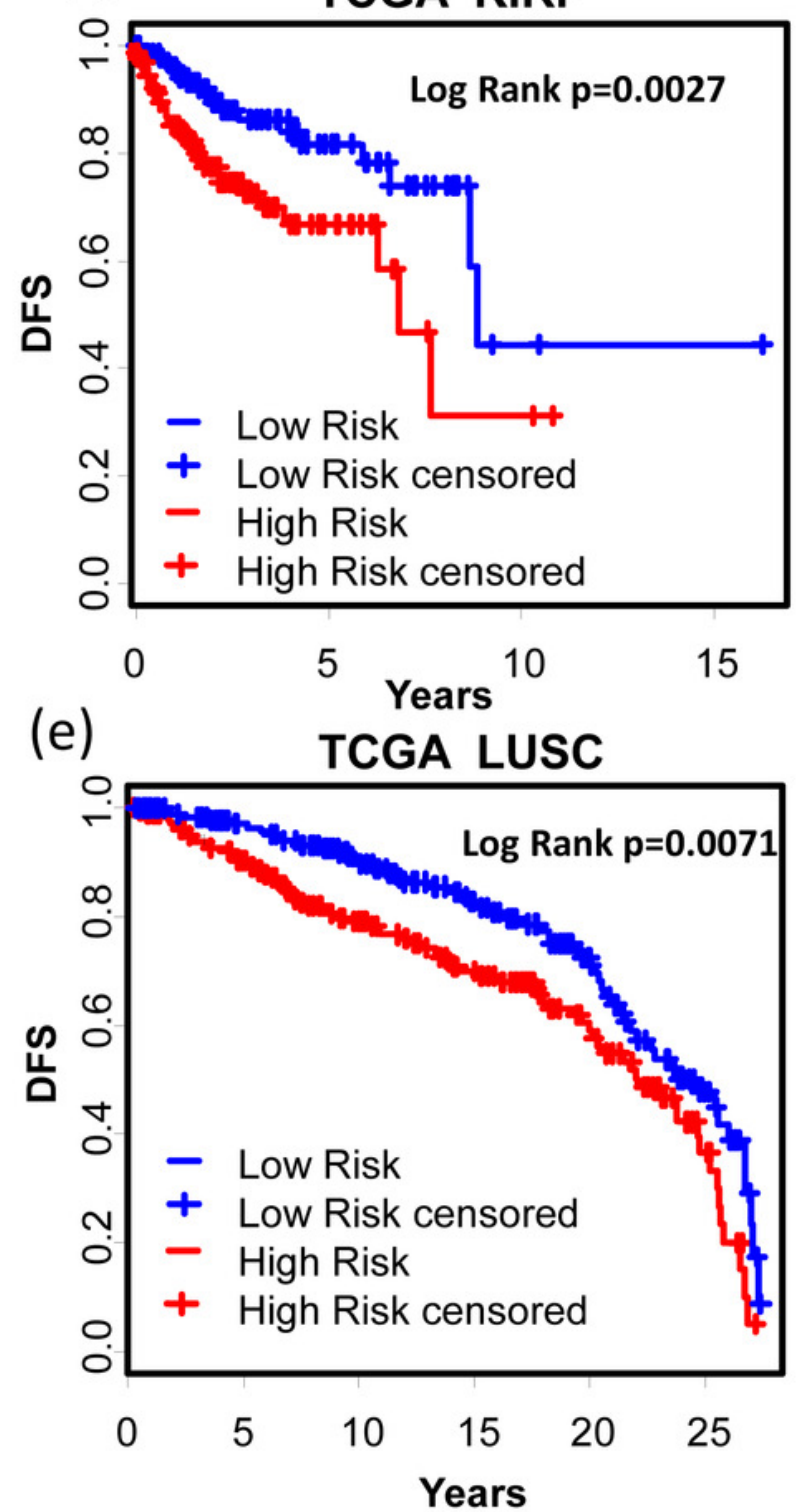

(b)

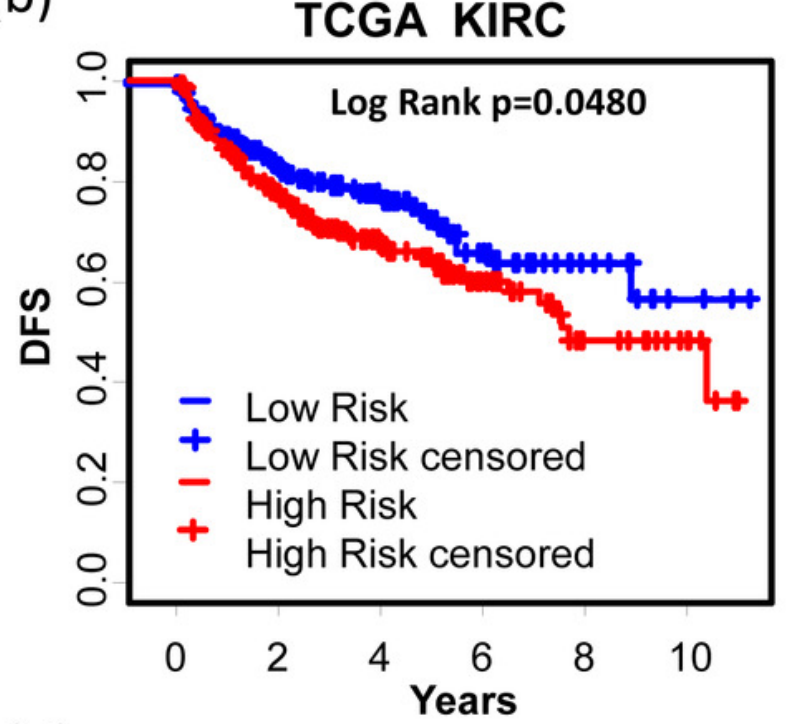

(d)

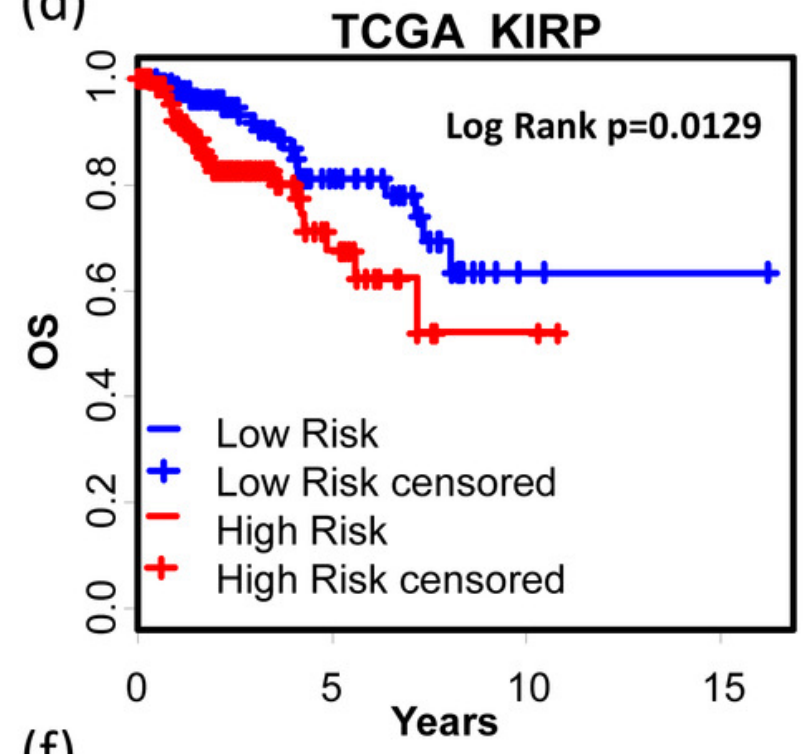

(f)

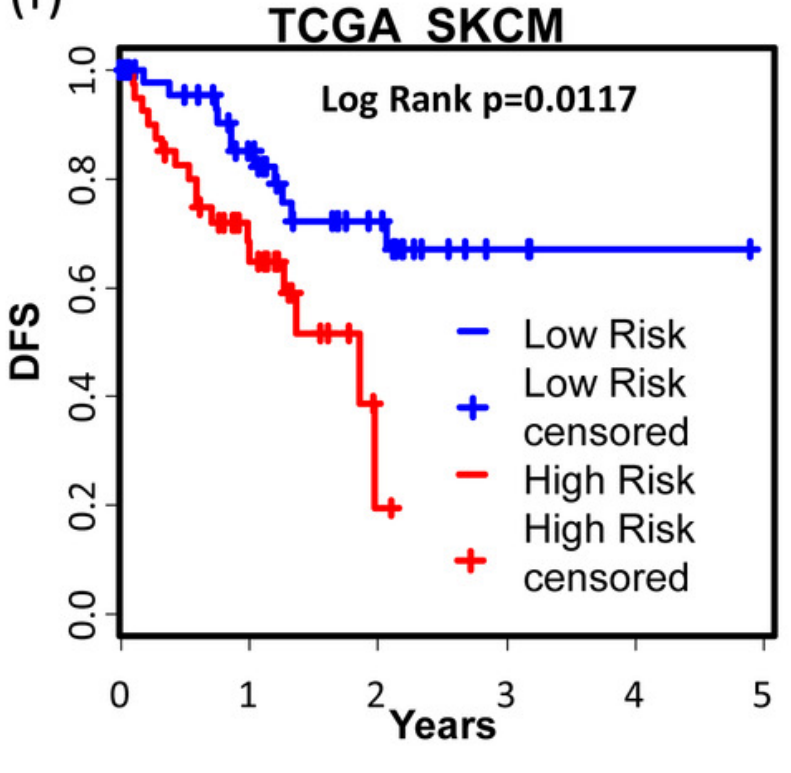




\section{Figure 8}

Kaplan-Meier analysis of the high and low 12-gene risk score patients for the major outcomes in other cancer types.

(a) OS in brain lower grade glioma (LGG). (b) OS in uveal melanoma (UVM). (c) OS in glioblastoma (GBM). (d) OS in cervical and endocervical cancers (CESC). (e) OS in pancreatic adenocarcinoma (PAAD). (f) OS in stomach adenocarcinoma (STAD).OS, overall survival. 
(a)

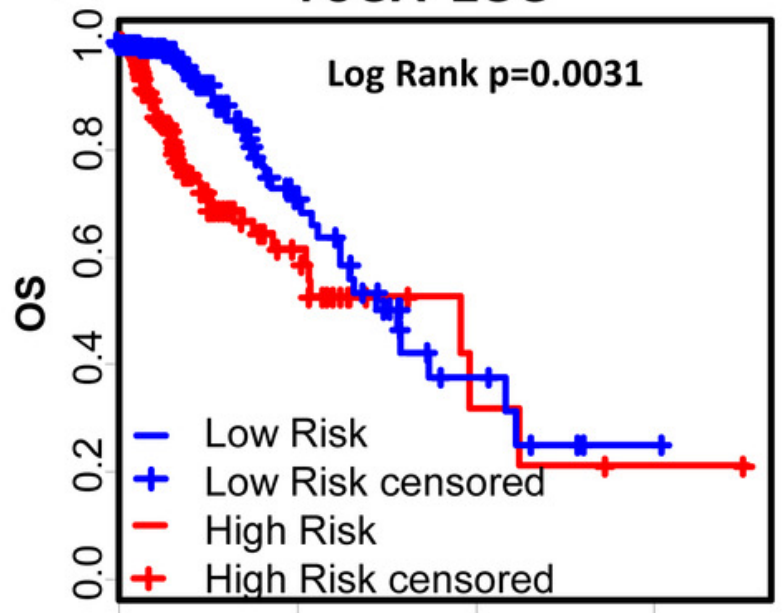

(c)

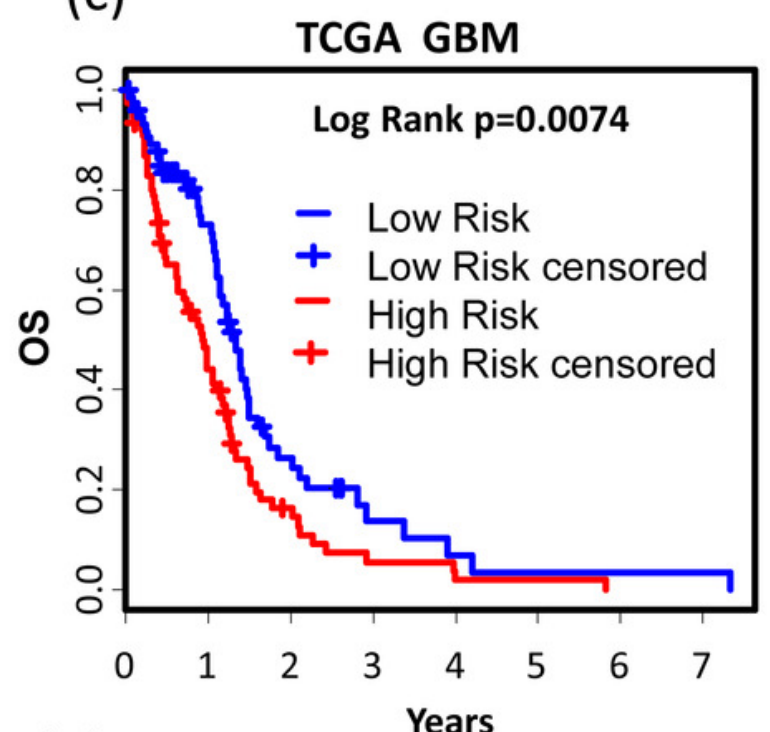

(e)

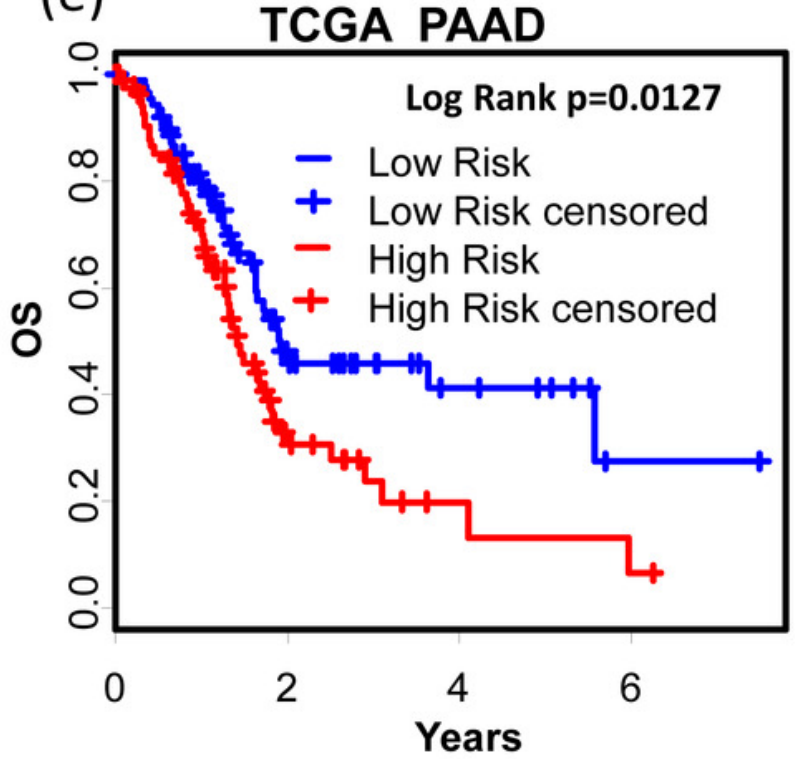

(b)

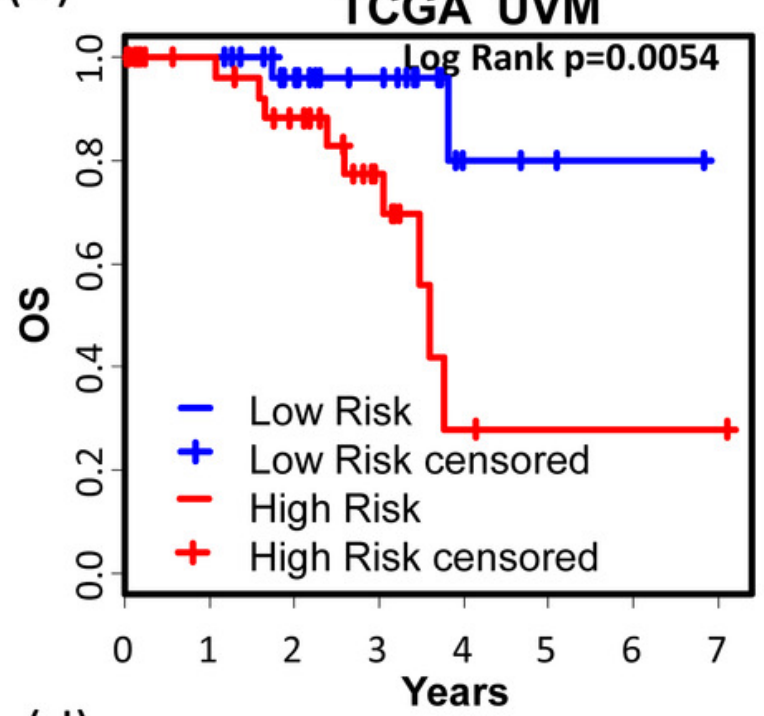

(d)

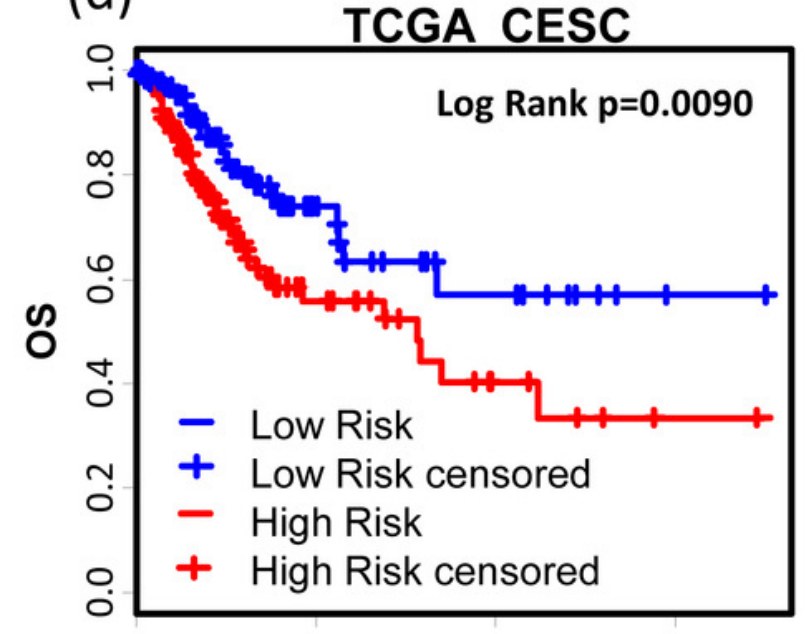

(f)

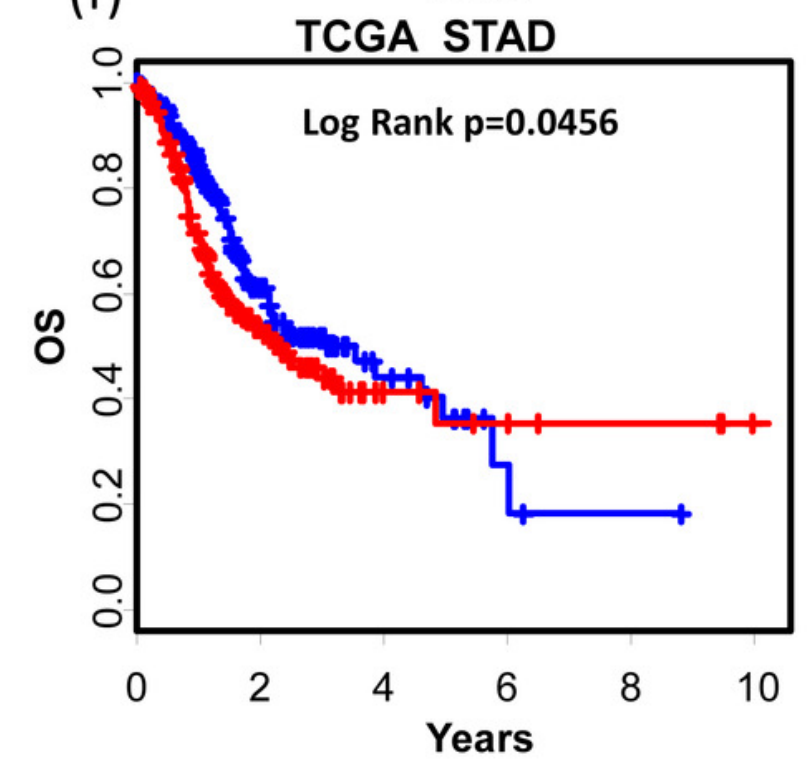


Table $\mathbf{1}$ (on next page)

Clinicopathologic features of 240 TCGA COAD patients. 


\begin{tabular}{|c|c|c|c|}
\hline Characteristic & $\begin{array}{c}\text { Training set }(\mathrm{N}=119) \\
\text { No. of patients }(\%)\end{array}$ & $\begin{array}{l}\text { Testing set }(\mathrm{N}=121) \\
\text { No. of patients }(\%)\end{array}$ & $p$ value \\
\hline Age $($ mean \pm SD) & $66.4 \pm 13.0$ & $63.2 \pm 13.8$ & $0.069^{\dagger}$ \\
\hline \multicolumn{4}{|l|}{ Gender } \\
\hline Male & $60(50.4 \%)$ & $54(44.6 \%)$ & \multirow{2}{*}{$0.438^{:}$} \\
\hline Female & $59(49.6 \%)$ & $67(55.4 \%)$ & \\
\hline \multicolumn{4}{|l|}{ Stage } \\
\hline I & $20(16.8 \%)$ & $20(16.5 \%)$ & \multirow{3}{*}{$0.998^{\S}$} \\
\hline II & $47(39.5 \%)$ & $48(39.7 \%)$ & \\
\hline III and IV & $52(43.7 \%)$ & $53(43.8 \%)$ & \\
\hline \multicolumn{4}{|l|}{ Primary tumor } \\
\hline $\mathrm{T} 1$ and $\mathrm{T} 2$ & $20(16.8 \%)$ & $23(19.0 \%)$ & \multirow{2}{*}{$0.737^{\ddagger}$} \\
\hline $\mathrm{T} 3$ and $\mathrm{T} 4$ & $99(83.2 \%)$ & $98(81.0 \%)$ & \\
\hline \multicolumn{4}{|c|}{ Microsatellite status } \\
\hline MSI-L & $23(19.3 \%)$ & $23(19.0 \%)$ & \multirow{3}{*}{$0.995^{\S}$} \\
\hline MSI-H & $20(16.8 \%)$ & $20(16.5 \%)$ & \\
\hline MSS & $76(63.9 \%)$ & $78(64.4 \%)$ & \\
\hline \multicolumn{4}{|c|}{ Lymphatic_invasion } \\
\hline No & $77(64.7 \%)$ & $77(63.6 \%)$ & \multirow{2}{*}{$0.999:$} \\
\hline Yes & $33(27.7 \%)$ & $34(28.1 \%)$ & \\
\hline Unknown & $9(7.6 \%)$ & $10(8.3 \%)$ & Excluded \\
\hline
\end{tabular}

1 tt test.

2 \#Fisher's exact test.

3 §Chi-squared test. 
Table 2 (on next page)

Statistics of the ROC analysis. 


\begin{tabular}{cccclc}
\hline Signatur & AUC & SE & Progressive $\boldsymbol{p}$ & \multicolumn{2}{c}{ Progressive 95\% CIs } \\
e & & & & Lower & $\begin{array}{c}\text { Upper } \\
\text { bound }\end{array}$ \\
\hline bound
\end{tabular}

Note. AUC: Area-Under-Curve;

SE: Standard Error.

95\% CIs: 95\% Confidence Intervals. 


\section{Table 3(on next page)}

Distribution of advanced stage patients between high- and low-score group. Fisher's exact test was used for statistical analysis. 


\begin{tabular}{lllll}
\hline \multicolumn{1}{c}{ Dataset } & \multicolumn{1}{c}{ Group } & Stage I\&II & Stage III\&IV & $\boldsymbol{p}$ value \\
\hline \multirow{2}{*}{ GSE17538 } & High score group & $19(20 \%)$ & $78(80 \%)$ & 0.0003 \\
& Low score group & $43(44 \%)$ & $54(56 \%)$ & \\
\multirow{2}{*}{ TCGA } & High score group & $53(49 \%)$ & $55(51 \%)$ & 0.0277 \\
& Low score group & $69(64 \%)$ & $38(36 \%)$ & \\
\hline
\end{tabular}

1 2 